\title{
The FUOR characteristics of the PMS star BN Orionis
}

\section{inferred from new spectroscopic and photometric observations ${ }^{\star}$}

\author{
V.S. Shevchenko ${ }^{1}$, O. Ezhkova ${ }^{1}$, H.R.E. Tjin A Djie ${ }^{2}$, M.E. van den Ancker ${ }^{2}$, P.F.C. Blondel ${ }^{2}$, and \\ D. de Winter ${ }^{2,3}$ \\ 1 Astronomical Institute of the Academy of Sciences of Uzbekistan, Astronomicheskaya 33, Tashkent 700052, Uzbekistan \\ 2 Astronomical Institute "Anton Pannekoek", University of Amsterdam, Kruislaan 403, 1098 SJ Amsterdam, The Netherlands \\ 3 Dpto. Física Teórica, C-XI, Facultad de Ciencias, Universidad Autónoma de Madrid, Cantoblanco, E-28049 Madrid, Spain
}

Received 4 June; accepted 7 October, 1996

Abstract. BN Ori is a young emission-line star with a peculiar light curve. During the first half of this century the star showed strong irregular brightness- variations, similar to those of Herbig Ae stars. In the current half of the century the light curve resembles that of a FU Ori-object (FUOR, after Ambartsumian 1971). It can be characterised by an initial large-scale rise in brightness followed by a gradual decay over a period of about 15 years. From various photometric patrol programmes we concluded that the star remained at the same brightness level for the last 30 years. Between 1991 and 1995 the spectrum of BN Ori was intensively observed and was found to exhibit some unique pecularities. We have analysed the visual spectra obtained with the $6 \mathrm{~m}$ BTA telescope of the SAO, the $60 \mathrm{~cm}$ and $48 \mathrm{~cm}$ telescopes at Mt. Maidanak, the $1.4 \mathrm{~m}$ $\mathrm{CAT}$ and the $1.5 \mathrm{~m}$ telescope of ESO and the UV-spectra obtained with the IUE in 1984 and 1986. The spectra show certain similarities with those of classical FUORs, such as a large-scale thermal stratification, with Balmer lines showing A6 - A7 spectral type wings, while other lines are typical for late F-type stars. However, in contrast to classical FUORs, BN Ori is not of high luminosity-class.

On the other hand the spectrum also shows similarities with those of Herbig A7e stars, although these stars have much stronger absorption lines in the UV from their outer shell and their circumstellar dust excesses are much larger than for BN Ori. Also, the rotation rate $v \sin i$ of BN Ori is between 180 and $220 \mathrm{~km} \mathrm{~s}^{-1}$, which is typical for A-type stars of $2-5 M_{\odot}$. This may indicate that BN Ori is a

Send offprint requests to: P.F.C. Blondel

* Based on observations collected at/with:

- Mt. Maidanak Observatory, Samarkand, Uzbekistan,

- Special Astrophysical Observatory, Russian Academy of

Sciences, Karachai-Cherkessia, Zelenchuk region, NizhniiArkhyz,

- European Southern Observatory, La Silla, Chile,

- Kitt Peak National Observatory, Tucson AZ, U.S.A.,

- Dutch Astronomical Station Ausserbinn, Switzerland,

- International Ultraviolet Explorer. fast rotating FUOR with an intermediate-mass precursor on which the outburst had a different effect than in the case of the classical FUORs (which are rotating slower and have a low-mass $\mathrm{T}$ Tauri star as precursor). In the BN Ori outburst most of the massive gas- and dust shell, characteristic for Herbig Ae stars, seems to have been removed, except for the $\mathrm{H} \alpha$ - and $\mathrm{Mg}$ II-emission region close to the photosphere. The disappearance of the circumstellar dust shell may explain the drastic variability-change in the light curve of BN Ori. The mass-accretion rates of BN Ori and several Herbig A7e stars (derived from the analysis of their UV-spectra in terms of disc-accretion) suggest that the FUOR outburst in BN Ori was due to a thermal runaway (Bell 1994) in its inner accretion-disc which was triggered by a modest increase in the accretion rate.

Key words: circumstellar matter — stars: emissionline - stars: BN Ori — stars: pre-main-sequence

\section{Introduction}

BN Ori (HD 245465, $\mathrm{BD}+06^{\circ} 971$ ) is an emission-line star about $3^{\circ}$ South-East to the $\lambda$ Ori cluster and probably connected to the star-formation region RSF1 in the Orion B complex by a dark lane of dust. A detailed review of the star's properties and the observational history up to 1989 has been given by Shevchenko (1989). Because redsensitive photographic plates for astronomical use did not become widely available until the fifties, the $(\mathrm{H} \alpha)$ emission character of BN Ori during the first half of the century cannot be ascertained at present. Nevertheless, before 1947 the star had several characteristics of the class which is presently known as the Herbig Ae stars: spectral type A7 (Cannon 1931) and, similar to T Ori (Beyer 1937), strong and irregular variations in brightness and colour, characteristic for the variability of circumstellar dust extinction. Around 1947 its variability character changed (Fig. 1): the amplitude of the large brightness-variations 
decreased and, after a large-scale rise of $\sim 0{ }^{\mathrm{m}} 5$ during the next 6 months, the brightness slowly decreased until it settled down at $V^{\mathrm{J}} \approx 9 .^{\mathrm{m}} 6$ around 1965. Since then the brightness remained at this level, apart from a small and short variation in 1991. The photometric history and the photometric observations made during the last decade are summarised in Sect. 2.

Table 1. Log of photometric observations of BN Ori, NN denotes the number of nights

\begin{tabular}{|c|c|c|c|c|}
\hline $\begin{array}{l}\text { Photometric } \\
\text { system }\end{array}$ & $\begin{array}{l}\text { Observatory } \\
+ \text { Telescope }\end{array}$ & Dates & $\mathrm{NN}$ & Observers \\
\hline $\begin{array}{l}\text { Walraven } \\
W U L B V \\
\text { (Table 2) }\end{array}$ & $\begin{array}{l}\text { ESO } \\
\text { Dutch - } 0.9 \mathrm{~m}\end{array}$ & $\begin{array}{l}\text { Feb'85 } \\
\text { Dec'85 } \\
\text { - Jan'86 } \\
\text { Nov'86 }\end{array}$ & $\begin{array}{l}2 \\
8\end{array}$ & $\begin{array}{l}\text { JK, ED } \\
\text { YN } \\
\text { JP }\end{array}$ \\
\hline $\begin{array}{l}\text { Strömgren } \\
u v b y\end{array}$ & $\begin{array}{l}\text { ESO } \\
\text { SAT }-0.5 \mathrm{~m}\end{array}$ & $\begin{array}{l}\text { Jan'85 } \\
\text { - Jan'90 }\end{array}$ & 62 & $\begin{array}{l}\text { LTPV } \\
\text {-group }\end{array}$ \\
\hline $\begin{array}{l}\text { Johnson/ } \\
\text { Cousins } \\
U B V(R I)_{\mathrm{C}} \\
\text { (Table 3) }\end{array}$ & $\begin{array}{l}\text { DASA - } 0.4 \mathrm{~m} \\
\text { ESO }-0.5 \mathrm{~m} \\
\text { DASA }-0.4 \mathrm{~m} \\
\text { KPNO- } 0.9 \mathrm{~m} \\
\text { DASA }-0.4 \mathrm{~m} \\
\text { DASA }-0.4 \mathrm{~m} \\
\text { ESO }-0.5 \mathrm{~m}\end{array}$ & $\begin{array}{l}\text { Nov'84 } \\
\text { Nov'86 } \\
\text { Nov'86 } \\
\text { Nov'86 } \\
\text { Nov'89 } \\
\text { Nov'92 } \\
\text { Jan'94 }\end{array}$ & $\begin{array}{r}5 \\
1 \\
11 \\
6 \\
1 \\
1 \\
1\end{array}$ & $\begin{array}{l}\text { ABl, YN, HC } \\
\text { GC, PL } \\
\text { HG, FI } \\
\text { ABr, CA } \\
\text { TM, CK } \\
\text { FM } \\
\text { FM }\end{array}$ \\
\hline $\begin{array}{l}\text { Johnson } \\
U B V R \\
\text { (Table 12) }\end{array}$ & $\begin{array}{l}\text { Mt. Maidanak } \\
0.5 \mathrm{~m}, 0.6 \mathrm{~m}\end{array}$ & $\begin{array}{l}\text { Dec'76 } \\
\text { - Sep'95 }\end{array}$ & 155 & $\begin{array}{l}\text { ROTOR } \\
\text {-group }\end{array}$ \\
\hline $\begin{array}{l}\text { ESO } \\
J H K L \\
\text { (Table 4) }\end{array}$ & ESO $-1 \mathrm{~m}$ & Feb'86 & 2 & $\mathrm{HC}$ \\
\hline
\end{tabular}

\begin{tabular}{|c|c|c|c|}
\hline \multicolumn{2}{|c|}{ Observers are: } & GC & G. Carrasco \\
\hline $\mathrm{ABl}$ & - A. Blom & $\mathrm{HC}$ & H. Cuypers \\
\hline $\mathrm{ABr}$ & A. Brown & HG & H. Grootjen \\
\hline CA & - C. Ambruster & JK & J.P.M. Koninx \\
\hline CK & - $\quad$ C. Koulis & JP & J.W. Prein \\
\hline ED & - $\quad$ E.M.F. Damen & PL & P. Loyola \\
\hline FI & F. Inklaar & TM & T. Meinen \\
\hline FM & F.J. Molster & YN & Y.K. Ng \\
\hline
\end{tabular}

Shevchenko (1989) was the first to discuss the possibility to interpret the light curve of BN Ori after 1945 in terms of a process related to the large-scale lightvariations of classical FUORs. The phenomenon of FUOR (Herbig 1977), although a prominent eruptive event of great intensity and duration, cannot be predicted nor explainded by the present evolution models (e.g. Palla \& Stahler 1992, 1993) of pre-main-sequence stars. At the same time, more and more young non-stationary stars exhibiting high-amplitude eruptions can be considered as related to FUORs. Herbig (1977) selected a group of stars (EX Lup, UZ Tau and VY Tau) showing recurrent brightening with great intensity and duration and later labelled them "EXORs" by the name of its brightest member EX Lup. In the early sixties, DR Tau, the most active classical T Tauri star (CTTS), exhibited a progressive brightening lasting for more than 15 years (Götz 1980). Some eruption related with those of FUORs were found from the light curves and the spectra of $\mathrm{Z} \mathrm{CMa}$
(Hartmann et al. 1989), V1331 Cyg and other stars which differ widely from classical FUORs.

The large-scale light curve of BN Ori as well as several spectral peculiarities indicate a certain similarity of the BN Ori properties with those of the FUORs. In this paper we first discuss the large-scale historical light curve of BN Ori (Sect. 2).

During the last decade we have obtained several highand low-resolution spectra of BN Ori in the visual and in the UV. A description of these spectra and a discussion of the peculiar spectral features is given in Sects. 4.2, 4.3 and 5 . The analysis of the optical spectra reveals a noticeable thermal stratification of the envelope of BN Ori, which is similar to what has been deduced from the analysis of the spectra of classical FUORs on the descending branch of their light curves. In BN Ori the stratification is reflected by the different values for the full-width at half maximum (FW hereafter) for lines from different temperature regions in the envelope. This can be interpreted by differential rotation in a rotationally flattened envelope or disc. Another similarity with classical FUORs is the high windvelocity measured from the fast variable (on time-scale of 1 day or less) P-Cygni type $\mathrm{H} \alpha$ and $\mathrm{Mg}$ II h\&k profiles of BN Ori. This type of outflow can probably only be driven by strong magnetic fields, e.g. produced in the neighbourhood of an accretion-disc boundary layer. Nevertheless, there are also differences between the spectrum of BN Ori and those of classical FUORs. Such differences may not be surprising in view of the fact that BN Ori is more massive $\left(\sim 2 M_{\odot}\right)$ and is rotating much faster than the classical FUORs.

We suggest that BN Ori in its present phase is the result of FUOR type outburst in a strongly variable Herbig A6 - 7e star. A similar transition was observed in 1983 for the Herbig A7e star V351 Ori (HD 38238), which changed from a strongly variable phase into a nonvariable A7e phase (van den Ancker et al. 1996), demonstrating that such phenomena are not exceptional for Herbig Ae stars. If this also happened with BN Ori, it becomes of interest to compare its spectral features with those of a Herbig A6 - 7e star as such a star might be similar to BN Ori before the FUOR outburst. We made a detailed comparison with the spectrum of HR 5999 (V856 Sco), a bright and strongly variable Herbig A5 7e star, which has been intensively studied during the last two decades (Bessell \& Eggen 1972; Tjin A Djie et al. 1989; Baade \& Stahl 1989). BN Ori has most lines in common with HR 5999, although the line-widths are partly different. However, it appears that the lines from the cool outer parts of the envelope (shell) are much fainter in BN Ori than in HR 5999, as is indicated by the smaller values of the equivalent widths (EW hereafter) listed in Table 8. This is consistent with the remarkable absence of circumstellar components of the NaID lines in the high-resolution spectra of BN Ori and the negligeable near-infrared excess (NIRE) and colour-excess in the 
spectral energy distribution (SED) of BN Ori. This comparison suggests that the FUOR outburst in BN Ori has removed its outer envelope almost completely, except for an optically-thin inner part where $\mathrm{H} \alpha$ is formed.

In Sect. 6 we summarise the similarities between the spectral features of BN Ori and those of the classical FUORs and the Herbig Ae stars. We also suggest a cause for the outburst in BN Ori, based on the mass-accretion rates of BN Ori, HR 5999 and BF Ori (A7e), which have been derived from the analysis of their low-resolution UVspectra taken with IUE (Blondel \& Tjin A Djie 1994, 1996).

\section{Photometry}

\subsection{History}

The variable character of BN Ori was detected by Cannon (1919) and since then the star has been monitored extensively both visually and photographically. Tsesevich made nearly $4000 m_{\mathrm{pg}}$ estimates of BN Ori from sky patrol photographic plate collections of the Harvard, Odessa, Sonneberg and Dushanbe observatories and constructed a light curve of $m_{\mathrm{pg}}$ over the period $1898-1964$ (Dragomiretskaya 1965; Tsesevich \& Dragomiretskaya 1973).

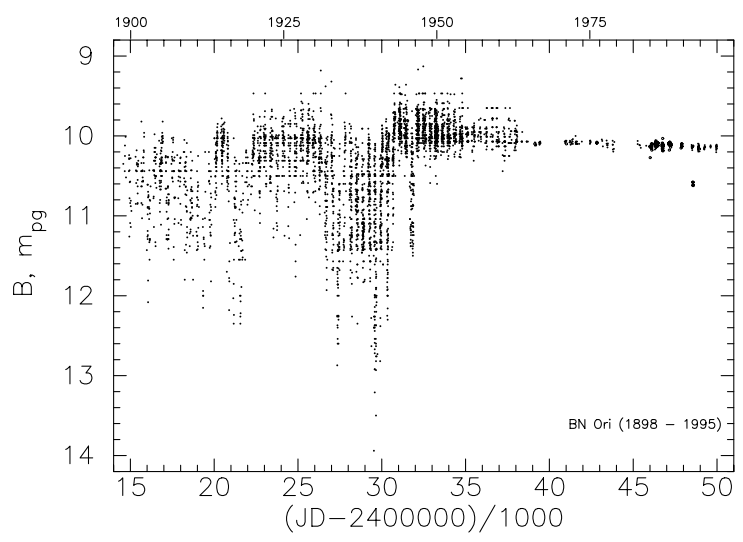

Fig. 1. Lightcurve of $m_{\mathrm{pg}}$ for BN Ori over the period 1898 - 1964, constructed by Tsesevich \& Dragomiretskaya (1973), and extended up to 1995

This light curve of BN Ori shows quiet periods with maximum brightness $m_{\mathrm{pg}} \sim 99^{\mathrm{m}} 7$, during which the brightness frequently decreases by at most 0.5 , separated by episodes of roughly 12.5 years, during which the mean brightness dropped with $1^{\mathrm{m}}$ or $2^{\mathrm{m}}$ over $100-200$ days or with $3^{\mathrm{m}}$ over $20-50$ days. Superposed on this longterm variability are rapid fluctuations with amplitude of 1. 5 during about 10 days. It was already noted by Beyer (1937) and confirmed by Hoffmeister (1949) and PayneGaposhkina (1952) that the behaviour of BN Ori is reminiscent of that of the RW Aur variables T Ori and XY Per
Table 2. Photometric Data of BN Ori on the Walraven $(W U L B V)$ system

\begin{tabular}{ccccccc}
\hline RJD $^{\#}$ & $V$ & $V-B$ & $B-U$ & $U-W$ & $B-L$ & $V^{\mathrm{J}}$ \\
\hline 111.576 & -1.117 & 0.197 & 0.402 & 0.200 & 0.228 & 9.66 \\
122.591 & -1.105 & 0.207 & 0.399 & 0.201 & 0.230 & 9.63 \\
428.642 & -1.111 & 0.205 & 0.411 & 0.200 & 0.233 & 9.64 \\
430.630 & -1.119 & 0.199 & 0.402 & 0.199 & 0.222 & 9.66 \\
436.617 & -1.111 & 0.198 & 0.405 & 0.195 & 0.228 & 9.65 \\
442.621 & -1.125 & 0.194 & 0.408 & 0.199 & 0.230 & 9.68 \\
443.627 & -1.119 & 0.198 & 0.403 & 0.193 & 0.221 & 9.67 \\
450.665 & -1.108 & 0.201 & 0.408 & 0.195 & 0.228 & 9.64 \\
451.631 & -1.107 & 0.202 & 0.406 & 0.194 & 0.224 & 9.64 \\
452.680 & -1.116 & 0.202 & 0.405 & 0.203 & 0.233 & 9.66 \\
745.763 & -1.124 & 0.201 & 0.401 & 0.195 & 0.226 & $9.67^{*}$ \\
\hline error: & 0.006 & 0.003 & 0.005 & 0.006 & 0.005 & 0.02 \\
\hline \# JD-2446000 & & & * simultaneous with IUE.
\end{tabular}

Table 3. Photometric Data of BN Ori on the Johnson/Cousins $U B V(R I)_{\mathrm{C}}$ system. The last column $(\mathrm{N}, \mathrm{O})$ gives the number of observations per night and the observatory: DASA(D), $\mathrm{ESO}(\mathrm{E})$ or Kitt Peak(K)

\begin{tabular}{lcccccc}
\hline RJD\# & $V$ & $B-V$ & $U-B$ & $V-R_{\mathrm{C}}$ & $V-I_{\mathrm{C}}$ & $\mathrm{N}, \mathrm{O}$ \\
\hline 6001.549 & 9.69 & 0.44 & 0.17 & 0.32 & 0.58 & $4, \mathrm{D}$ \\
6002.525 & 9.65 & 0.48 & 0.20 & 0.29 & 0.59 & $4, \mathrm{D}$ \\
6005.575 & 9.67 & 0.47 & 0.19 & 0.30 & 0.58 & $5, \mathrm{D}$ \\
6006.546 & 9.64 & 0.49 & 0.17 & 0.29 & 0.57 & $5, \mathrm{D}$ \\
6007.584 & 9.86 & 0.41 & 0.18 & 0.24 & 0.54 & $4, \mathrm{D}$ \\
6731.596 & 9.70 & 0.46 & - & 0.29 & 0.58 & $3, \mathrm{D}$ \\
6739.917 & 9.66 & 0.45 & 0.14 & 0.29 & 0.59 & $1, \mathrm{~K}$ \\
6742.994 & 9.66 & 0.47 & 0.14 & 0.29 & 0.59 & $1, \mathrm{~K}$ \\
6743.823 & 9.62 & 0.46 & 0.13 & 0.30 & 0.60 & $1, \mathrm{~K}$ \\
6743.947 & 9.64 & 0.46 & 0.14 & 0.29 & 0.57 & $1, \mathrm{~K}$ \\
6745.427 & 9.67 & 0.44 & - & 0.31 & 0.58 & $3, \mathrm{D}$ \\
6747.494 & 9.64 & 0.46 & - & 0.27 & 0.56 & $3, \mathrm{D}$ \\
6747.801 & 9.68 & 0.47 & 0.16 & 0.29 & 0.57 & $1, \mathrm{E}$ \\
6748.836 & 9.65 & 0.45 & 0.14 & 0.29 & 0.60 & $1, \mathrm{~K}$ \\
6748.912 & 9.70 & 0.45 & 0.12 & 0.30 & 0.61 & $1, \mathrm{~K}$ \\
6754.766 & 9.66 & 0.47 & 0.14 & 0.28 & 0.59 & $1, \mathrm{~K}$ \\
6754.951 & 9.64 & 0.45 & 0.16 & 0.30 & 0.59 & $1, \mathrm{~K}$ \\
6759.433 & 9.76 & 0.42 & - & 0.31 & 0.59 & $3, \mathrm{D}$ \\
6760.433 & 9.61 & 0.47 & - & 0.25 & 0.58 & $3, \mathrm{D}$ \\
6762.490 & 9.68 & 0.45 & - & 0.29 & 0.58 & $3, \mathrm{D}$ \\
6763.528 & 9.56 & 0.47 & - & 0.28 & 0.56 & $3, \mathrm{D}$ \\
6764.445 & 9.69 & 0.43 & - & 0.29 & 0.58 & $3, \mathrm{D}$ \\
6765.424 & 9.71 & 0.44 & - & 0.30 & 0.55 & $3, \mathrm{D}$ \\
6766.463 & 9.66 & 0.45 & - & 0.29 & 0.59 & $3, \mathrm{D}$ \\
6767.444 & 9.65 & 0.46 & - & 0.28 & 0.57 & $3, \mathrm{D}$ \\
7854.516 & 9.66 & 0.49 & - & 0.25 & 0.52 & $3, \mathrm{D}$ \\
8936.646 & 9.69 & 0.47 & - & 0.31 & 0.43 & $1, \mathrm{D}$ \\
9371.616 & 9.62 & 0.52 & 0.09 & 0.30 & 0.58 & $1, \mathrm{E}$ \\
\hline error: & 0.01 & 0.01 & 0.02 & 0.01 & 0.02 & $\mathrm{E}, \mathrm{K}$ \\
& 0.04 & 0.02 & 0.02 & 0.02 & 0.02 & $\mathrm{D}$ \\
\hline \# JD-2440000 & & & $*$ & simultaneous with IUE.
\end{tabular}

and we can extend the similarity now to other Herbig Ae stars with Algol-type minima such as BF Ori, UX Ori, WW Vul and HR 5999. In a discussion of the photometric variability of BN Ori, Dragomiretskaya (1965) concluded that the large amplitude-variations cannot be due to temperature differences on the stellar surface, since a dimming 
Table 4. NIR photometric data of BN Ori

\begin{tabular}{ccccc}
\hline JD & $J$ & $H$ & $K$ & $L$ \\
\hline 2446486.5 & 8.74 & 8.41 & 8.33 & $>8.0$ \\
2446488.5 & 8.68 & 8.36 & 8.25 & $>8.0$ \\
\hline error: & 0.08 & 0.08 & 0.06 & - \\
\hline
\end{tabular}

by 1.5 would already require a full coverage by cool spots of the stellar surface. Dragomiretskaya also used the visual photometry by Esch (1925), Beyer (1937) and Martinov (1951), to derive the colour indices for a number of dates and the resulting colour-magnitude diagram shows that the strong variations of BN Ori follow a reddening-line similar to those followed by many Herbig Ae stars. Such a type of variation is usually ascribed to obscuration by variable amounts of circumstellar dust (Wenzel 1968; Wenzel et al. 1971). For BN Ori the slope of the reddening-line was about 3, which is close to that of interstellar dust. Tsesevich \& Lange observed that BN Ori showed Algollike variations with a period of 27 days (Martinov 1951). From an analysis of quasi-periodic features during highactivity episodes in the light curve of BN Ori, Ishchenko (1982) detected a quasi-cyclic 18 - 22 day periodicity superimposed on a stochastical behaviour and attributed this periodicity to the rotation of obscuring circumstellar dust clouds.

Figure 1 shows that the large-scale structure of the light curve changed around 1940: the amplitude of the strong variations decreased and the brightness maximium increased to $m_{\mathrm{pg}} \approx 9 \mathrm{~g}^{\mathrm{m}} 2$ in Dec'46. Since then the variations were gradually quenched from an amplitude of $0^{\mathrm{m}} 4$ to about $0^{\mathrm{m}} 1$ in 1966 , while the maximum of the brightness-variations slowly decreased to $m_{\mathrm{pg}} \sim 10{ }^{\mathrm{m}} 1$. In Sect. 6 we shall compare this behaviour with those of classical FUORs. After 1965 all observations have been made photoelectrically. Between 1965 and 1975 Zajtseva (1968, 1970), Kolotilov \& Zajtseva (1976) and Kolotilov et al. (1977) have made several observations of BN Ori on the $U B V$ and $J H K L$ photometric systems, but they did not detect a pronounced variation.

\subsection{Photometry since 1976}

The star has been observed regularly by various observers on several photometric systems (see Table 1).

1. Between 1976 and $1995 U B V R$ observations were obtained at the Mt. Maidanak observatory near Samarkand (Table 12). This was done in the course of the ROTOR (Research of Traces of Rotation) photometric program with a single-channel photometer mounted on the $60 \mathrm{~cm}$ Zeiss reflector and the $48 \mathrm{~cm}$ $\mathrm{AZT}^{1} 14$ reflector (Kilyachkov \& Shevchenko 1977).

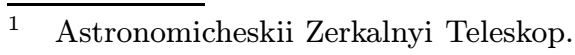

The data of JD 2448582 are simultaneous with the high-resolution spectroscopy at SAO (Sect. 4.3)

2. From 1985 to 1990 BN Ori was observed on the Strömgren ubvy system by the LTPV-group (Long Term Photometry of Variables) of ESO observers, with the Strömgren Automatic Telescope (SAT; formerly known as the Danish $50 \mathrm{~cm}$ telescope) at La Silla. This telescope is equipped with a simultaneous multicolour photometer. Any systematic effects due to nonsimultaneous measurements at the different passbands are therefore avoided. Since these observations have already been published (Sterken et al. 1993, 1995), they are not listed in this paper.

3. Photometric data on the Walraven $W U L B V$ system were obtained during 3 observing runs in 1985 and 1986 with the Dutch Light Collector at ESO, La Silla. Measuring and reduction procedures have been explained by Lub \& Pel (1977). During these observations the Walraven $W U L B V-\log _{10}$ intensity values were measured simultaneously in the different passbands. The results of these measurements are listed in Table 2, where the last column gives the corresponding magnitude on the Johnson system calculated with the formula suggested by Brand \& Wouterloot (1988).

4. Optical photometry of BN Ori, on the Johnson/Cousins $U B V(R I)_{\mathrm{C}}$ system, has been obtained in 1986 and 1994 with the ESO $50 \mathrm{~cm}$ telescope at La Silla, in Nov' 86 with the KPNO $90 \mathrm{~cm}$ telescope at Kitt Peak and on several occasions between 1984 and 1992 with the $40 \mathrm{~cm}$ telescope at the Dutch Astronomical Station at Ausserbinn (DASA), Switzerland. The results of these measurements are collected in Table 3 and the data of JD 2446747 were obtained simultaneously with the high-resolution IUE observation of BN Ori.

5. Since 1984 BN Ori has also been observed on the $U B V(R I)_{\mathrm{C}}$ system by W. Herbst and collaborators in the course of a monitoring program at the "Van Vleck" observatory (private communication).

6. NIR photometric data on the ESO JHKL system (Bouchet et al. 1989, 1991), of BN Ori were obtained in Feb'86 with the ESO $1 \mathrm{~m}$ telescope at La Silla. During these observations, the telescope was equipped with an InSb detector with a $15^{\prime \prime}$ diaphragm. Sky subtraction was achieved by chopping with a frequency of $8 \mathrm{~Hz}$ in the east-west direction with a throw of $30^{\prime \prime}$ amplitude. The results are given in Table 4.

After transformation of the data on the various systems to the Johnson magnitude $V^{\mathrm{J}}$ and colour $(B-V)^{\mathrm{J}}$ we can hardly find any change in brightness and colour over these years. The average values are: $V^{\mathrm{J}}=9 \cdot^{\mathrm{m}} 65 \pm 0.03$ and $(B-V)^{\mathrm{J}}=0{ }^{\mathrm{m}} 47 \pm 0 .{ }^{\mathrm{m}} 02$. Only on one occasion (Oct. 29 - Nov. 2, 1991), the star seemed to dim by 0.m 5 (Kovalchuk 1991). Because there are no data available for the 34 nights preceding Oct. 29 and for the 12 nights following Nov.2, we can only state that the variation was limited to at most 


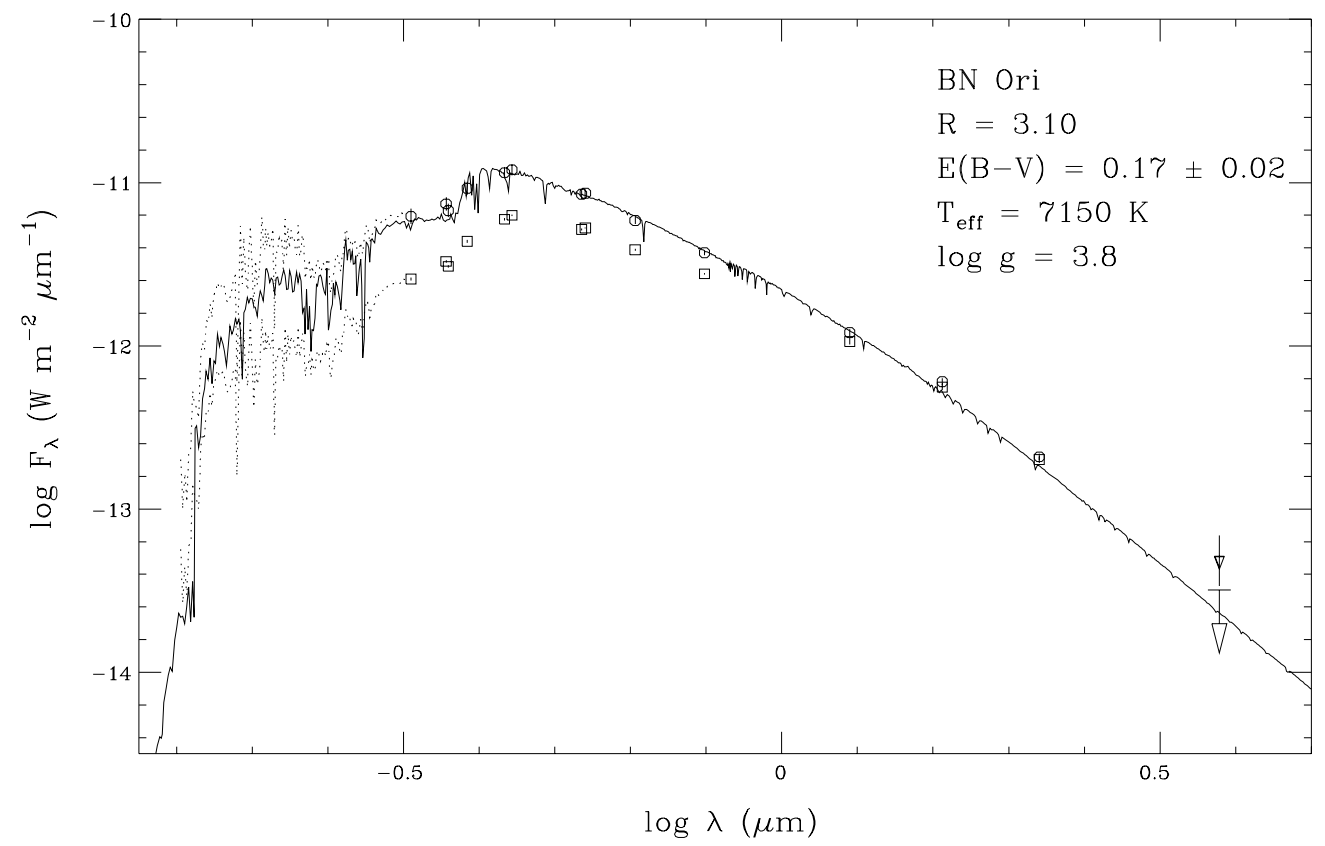

Fig. 2. Observed (squares) and extinction-corrected (circles) SED of BN Ori based on the data of Feb' 86 . The $L$-band observation of Cohen (1973) is indicated by the "upper" triangle (with error-bar). The $L$-band observation from Table 4 is indicated by the "lower" triangle (with upperlimit). The solid line is the Kurucz model of $T_{\text {eff }}=7150 \mathrm{~K}, \log g=3.8$ and solar abundance, normalised to the extinction-corrected $V$-band flux of BN Ori. The dotted lines are the observed- and extinction-corrected UV-fluxes

51 nights. The variation is along the reddening-line with slope 3 , so that this event could have been due to infalling circumstellar dust which evaporated in the neighbourhood of the star.

Since 1976 we have obtained only two observations in the NIR (Table 4), and in Fig. 2 we give a SED of BN Ori based on these data of Feb'86. After transformation of the magnitudes to fluxes and correction of these fluxes for foreground extinction with the law of Savage \& Mathis (1979) and $E(B-V)=0$ m 07 (Sect. 4.3), the corrected SED could be fitted with a Kurucz (1991) model for $T_{\text {eff }}=7150 \mathrm{~K}$ and $\log g=3.8$, corresponding to a spectral type of F0 III - IV (Sect. 5) and an $E(B-V)=0$ m 17 . From Fig. 2 we note that we get a good agreement in the visual and NIR wavelength ranges, but that there remains an excess in the UV. This excess will be explained (Sect. 5) in terms of the contribution from a (hot) boundary layer between the star and accretion-disc. If we compare our NIR data of 1986 with those obtained before 1977 (Glass \& Penston 1974; Kolotilov et al. 1977) we note no difference in the $H$ and $K$ magnitudes. However, our $J$ band flux is somewhat larger than that of Kolotilov et al. and our $L$-band flux is significantly smaller than the one Cohen $\left(1973 ; L \approx 7^{\mathrm{m}} 6\right)$ measured in 1971. The earlier attempts to match the SED (Kolotilov \& Zajtseva 1976; Brown et al. 1986) were based on this higher $L$-band flux. No IRAS point-source was detected at the position of
BN Ori, probably because the far-IR fluxes of the star are not higher than the background.

From the Walraven photometry (which has two passbands in the UV: $W$ at $3236 \AA$ and $U$ at $3623 \AA$ ), we can get a measure of the magnitude of the Balmer discontinuity $\left(D_{\mathrm{B}}\right)$. The mean of the 9 observations of Dec' 85 - Jan' 86 gives $D_{\mathrm{B}}=0 .{ }^{\mathrm{m}} 80$. This is a deficiency of $0{ }^{\mathrm{m}} \cdot 48$ with respect to the prediction of the Kurucz (1991) model for $T_{\text {eff }}=7200 \mathrm{~K}$ and $\log g=3.5$ which we take for the classification F0 III (Sect. 5). If this is due to the contribution of recombination of $\mathrm{H}^{+}$free-bound transitions near the star, we can derive (Garrison 1978) that for a temperature of $10^{4} \mathrm{~K}$ in this region the emission measure is $1.8610^{35} \mathrm{~cm}^{-5}$. If we assume that this ionosphere is in hydrostatic equilibrium with a scale-height of $4 R_{\star}$ we find within $2 R_{\star}$ an electron density $N_{\mathrm{e}}=3.3410^{11} \mathrm{~cm}^{-3}$.

For HR 5999 (A5 - 7 III) we used the Walraven photometry to derive a $D_{\mathrm{B}}$ equal to 1.23 which results in a deficiency of 0.20 when compared to a Kurucz (1991) model with $T_{\text {eff }}=8000 \mathrm{~K}$. This gives an emission measure of $6.010^{35} \mathrm{~cm}^{-5}$, which corresponds to $N_{\mathrm{e}}=5.610^{11} \mathrm{~cm}^{-3}$ close to the star, so that $N_{\mathrm{e}}^{2}$ is 2.8 times the value found for BN Ori.

\section{Imaging}

In the two-dimensional CCD spectrograms, of $\mathrm{H} \alpha$ taken in Jan'95, weak nebular emission-lines were detected along the spatial direction of the CCD image, which 
Table 5. Log of spectroscopic observations of BN Ori. Columns 8 to 10 denote the dispersion $\boldsymbol{D}\left(\AA \mathrm{mm}^{-1}\right)$ the resolution $\boldsymbol{R}$ $\left(\AA \mathrm{pix}^{-1}\right)$ and the exposure time $\Delta t(\mathrm{~min})$. The IUE imagenumbers are given in Col. 5 and the first digit denotes the used camera $(1=\mathrm{LWP}, 3=\mathrm{SWP})$ and LAP means large aperture. Observers: AB - A. Brown, DW - D. de Winter, HC - H. Cuypers, HT - H.R.E. Tjin A Djie, MA - M.E. van den Ancker, VK - V. Kotyshev, VS - V.S. Shevchenko

\begin{tabular}{|c|c|c|c|c|c|c|c|c|c|c|}
\hline $\begin{array}{c}\text { Spectral } \\
\text { Range }(\AA)\end{array}$ & Date & UT & RJD \# & $\begin{array}{l}\text { Observatory } \\
+ \text { Telescope }\end{array}$ & $\begin{array}{l}\text { Spect } \\
+\mathrm{D}\end{array}$ & $\begin{array}{l}\text { graph } \\
\text { ector }\end{array}$ & $D$ & $R$ & $\Delta t$ & Obs. \\
\hline $3700-7000$ & $12 / 11 / 83$ & $22: 25$ & 5651.4345 & Maidanak $0.6 \mathrm{~m}$ & UAGS & tube & 90 & 2.5 & 60 & VS \\
\hline $6000-8700$ & $13 / 11 / 83$ & $22: 48$ & 5652.4500 & Maidanak $0.6 \mathrm{~m}$ & UAGS & tube & 90 & 2.5 & 60 & VK \\
\hline $3800-7200$ & $24 / 02 / 86$ & $02: 15$ & 6485.5944 & $\mathrm{ESO} 1.5 \mathrm{~m}$ & $\mathrm{~B} \& \mathrm{C}$ & IDS & 172 & 1.7 & 2 & $\mathrm{HC}$ \\
\hline $3600-6790$ & $21 / 11 / 91$ & $19: 40$ & 8582.3195 & SAO BTA $6 \mathrm{~m}$ & MSS & A700y & 27 & 0.65 & 65 & VS \\
\hline $4180-7860$ & $07 / 12 / 92$ & $06: 11$ & 8963.7576 & $\mathrm{ESO} 1.5 \mathrm{~m}$ & $\mathrm{~B} \& \mathrm{C}$ & CCD & 129 & 1.80 & 8 & MA \\
\hline $6090-8720$ & $08 / 12 / 92$ & $04: 44$ & 8964.6972 & ESO $1.5 \mathrm{~m}$ & $\mathrm{~B} \& \mathrm{C}$ & $\mathrm{CCD}$ & 88 & 1.28 & 8 & MA \\
\hline $5858-5910$ & $16 / 12 / 94$ & $02: 27$ & 9702.6021 & ESO CAT $1.4 \mathrm{~m}$ & CES & CCD & - & 0.11 & 45 & MA \\
\hline $3700-7090$ & $16 / 12 / 94$ & $03: 58$ & 9702.6653 & $\mathrm{ESO} 1.5 \mathrm{~m}$ & $\mathrm{~B} \& \mathrm{C}$ & CCD & 114 & 1.59 & 6 & DW \\
\hline $5858-5910$ & $17 / 01 / 95$ & 01:49 & 9734.5757 & ESO CAT $1.4 \mathrm{~m}$ & CES & CCD & - & 0.11 & 45 & MA \\
\hline $6536-6591$ & $17 / 01 / 95$ & $02: 46$ & 9734.6153 & ESO CAT $1.4 \mathrm{~m}$ & CES & CCD & - & 0.12 & 45 & MA \\
\hline $7650-7715$ & $20 / 01 / 95$ & 01:08 & 9737.5474 & ESO CAT $1.4 \mathrm{~m}$ & CES & CCD & - & 0.14 & 45 & MA \\
\hline $6536-6591$ & $20 / 01 / 95$ & 02:05 & 9737.5867 & ESO CAT $1.4 \mathrm{~m}$ & CES & $\mathrm{CCD}$ & - & 0.12 & 45 & MA \\
\hline $1150-2000$ & $22 / 01 / 84$ & 10:04 & 5721.9819 & IUE LAP 322075 & Echelle & videcon & - & 7 & 180 & $\mathrm{HT}$ \\
\hline $1800-3200$ & $22 / 01 / 84$ & $13: 09$ & 5722.0521 & IUE LAP 102694 & Echelle & videcon & - & 7 & 12 & $\mathrm{HT}$ \\
\hline $1150-2000$ & $11 / 10 / 86$ & 06:09 & 6714.7979 & IUE LAP 329420 & Echelle & videcon & - & 7 & 120 & $\mathrm{AB}$ \\
\hline $1800-3200$ & $11 / 10 / 86$ & $08: 15$ & 6714.8472 & IUE LAP 109294 & Echelle & videcon & - & 7 & 10 & $\mathrm{AB}$ \\
\hline $1150-2000$ & $11 / 11 / 86$ & 04:08 & 6745.7451 & IUE LAP 329657 & Echelle & videcon & - & 7 & 210 & $\mathrm{HT}, \mathrm{AB}$ \\
\hline $1800-3200$ & $11 / 11 / 86$ & $07: 59$ & 6745.8361 & IUE LAP 109508 & Echelle & videcon & - & 7 & 10 & $\mathrm{HT}, \mathrm{AB}$ \\
\hline $1800-3200$ & $12 / 11 / 86$ & $19: 43$ & 6747.5924 & IUE LAP 109512 & Echelle & videcon & - & 0.2 & 780 & $\mathrm{HT}, \mathrm{AB}$ \\
\hline $1150-2000$ & $13 / 11 / 86$ & $10: 00$ & 6747.9792 & IUE LAP 329660 & Echelle & videcon & - & 7 & 190 & $\mathrm{HT}, \mathrm{AB}$ \\
\hline
\end{tabular}

\# JD-2440000.

demonstrates that BN Ori is surrounded by a very faint emission (reflection) nebula. This is another property which BN Ori shares with the class of Herbig Ae/Be stars. Since these weak lines extended over the entire CCD frame, the diameter of this nebula must be larger than that covered by this frame $\left(\sim 10^{\prime \prime}\right)$. In order to get more insight in the morphology of this nebula, CCD images of BN Ori through Bessell $U$ and $V$, Gunn $z$, and a narrowband $(\mathrm{FW}=13.5 \AA$, centered at $6558.88 \AA) \mathrm{H} \alpha$-filter (No. 674) were obtained in Feb'95 (de Winter 1995) with the Dutch $90 \mathrm{~cm}$ telescope at ESO. However, we did not detect nebulosity around BN Ori on any of these images, which may be caused by the fact that the bandwidth of the $\mathrm{H} \alpha$-filter is still too large. In these cases the contribution from other surrounding wavelengths may be too high to permit imaging of the nebulosity in $\mathrm{H} \alpha$.

\section{Visual spectroscopy}

\subsection{History}

The spectroscopic information on BN Ori from the period before 1960 is scarce. The first spectral classification of A7, was made by Cannon (1931) for the HDE catalogue, from objective prism spectra on plates exposed between 1925 and 1931. This classification was later confirmed by Parenago (1933), Hoffmeister (1949) and PayneGaposhkina (1952). Herbig (1954) estimated a type A5 A7 from a low-dispersion slit spectrogram, but from an additional spectrum $\left(\boldsymbol{D}=75 \AA \mathrm{mm}^{-1}\right.$, taken on Mar. 6, 1955), he noted that the spectrum of BN Ori is pecu- liar and that it may be composite with types A5 - A6 and mid-F9 (Herbig 1960a). In 1968 and 1969 Zajtseva (1971) took a number of blue-slit spectra $(\boldsymbol{D}=140-$ $250 \AA \mathrm{mm}^{-1}$ ) from which she classified the spectrum of BN Ori as F0. A microphotometer tracing in that paper shows absorption lines near 4525, 5163 and $5265 \AA$ (identified by her as Fe I), CaII K and Mg II at $4481 \AA$. From the slope of the continuum between 6000 and $4000 \AA$ a spectrophotometric temperature of $9700 \mathrm{~K}$ was derived, which is consistent with spectral type A7 - A9. Photoelectric spectral scans in the range $6600-3200 \AA$ made in Jan'75 allowed Kolotilov \& Zajtseva (1976) to determine the residual strengths of the $\mathrm{H} \gamma, \mathrm{H} \delta, \mathrm{H} \varepsilon$ and $\mathrm{Ca} I \mathrm{~K}$ lines, which led the authors to a classification F2 - F3. The only emission line in the visual spectrum of BN Ori is $\mathrm{H} \alpha$. Between Jul'71 and Dec'75, 18 spectrograms of $\mathrm{H} \alpha(\boldsymbol{D}$ $=20 \AA \mathrm{mm}^{-1}, \boldsymbol{R}=1 \AA \mathrm{pix}^{-1}$ ) were taken by Kolotilov \& Zajtseva (1976). The line profile of $\mathrm{H} \alpha$ in these spectra is double-peaked with an absorption trough at $-50 \mathrm{~km} \mathrm{~s}^{-1}$. Its blue emission-component varies strongly and has been observed to change into an absorption-component (with maximum velocity $-600 \mathrm{~km} \mathrm{~s}^{-1}$ ) within 24 .

\subsection{Low-dispersion spectra}

Since 1983 we have obtained a number of high-and lowresolution spectra of BN Ori. The observational details are listed in Table 5. We will compare the spectral information of BN Ori with that of FU Ori (Herbig 1966; Reipurth 1990) and that of the bright Herbig A5 - 7 IIIe 
Table 6. Identification, equivalent widths $(\AA)$, full widths at half maximum $\left(\mathrm{km} \mathrm{s}^{-1}\right)$ and source $(\boldsymbol{S})$ of the lines in the red part of the visual spectra of BN Ori, HR 5999 and FU Ori. See Table 10 for the description of the used symbols

\begin{tabular}{|c|c|c|c|c|c|c|c|c|c|c|c|c|c|}
\hline \multirow{2}{*}{$\begin{array}{l}\lambda_{0} \\
(\AA)\end{array}$} & \multirow[b]{2}{*}{ Line id. } & \multirow{2}{*}{$\begin{array}{c}\mathrm{E}_{\chi} \\
(\mathrm{eV})\end{array}$} & \multirow[b]{2}{*}{$S$} & \multicolumn{2}{|c|}{ BN Ori } & \multicolumn{5}{|c|}{ HR 5999} & \multicolumn{3}{|c|}{ FU Ori } \\
\hline & & & & EW & FW & EW & FW & $\mathrm{EW}^{5}$ & ${ }^{5} \mathrm{FW}^{6}$ & $\mathrm{EW}^{6}$ & EW & & FW \\
\hline \multirow[t]{2}{*}{5889.95} & $\mathrm{Na}$ I $\mathrm{D}_{2}$ & 0.00 & $\mathrm{~s}$ & $0.00^{1,8}$ & - & $0.38^{8}$ & 31 & 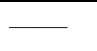 & - & & 2.53 & & $66:$ \\
\hline & & & d & $0.13^{1,8}$ & 400 & $0.37:^{8}$ & 250: & 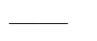 & - & & & & - \\
\hline \multirow[t]{2}{*}{5895.92} & $\mathrm{Na} I \mathrm{D}_{1}$ & 0.00 & $\mathrm{~s}$ & $0.00^{1,8}$ & - & $0.30^{8}$ & 28 & 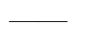 & - & 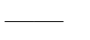 & 2.53 & 9 & $66:$ \\
\hline & & & d & $0.09^{1,8}$ & 400 & $0.35:^{8}$ & 250: & & - & & - & & - \\
\hline 6496.90 & Ba II & 0.60 & & $0.42^{7}$ & 402 & $0.44^{4}$ & 419 & $\longrightarrow$ & - & 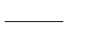 & - & & - \\
\hline 5562.82 & $\mathrm{H} \alpha$ & 10.20 & pcs & $-.80^{1,8}$ & - & $-3,-8^{4}$ & - & - & - & 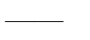 & - & & - \\
\hline 6707.89 & Li I & 0.00 & ps & $0.30^{7}$ & 244 & 0.0 & - & - & - & 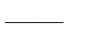 & 0.47 & 3 & - \\
\hline 7664.90 & K I (1) & 0.00 & $\mathrm{i}$ & $0.09:^{8}$ & - & - & - & $\longrightarrow$ & - & 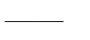 & - & & - \\
\hline 7698.96 & $\mathrm{~K}_{\mathrm{I}}$ & 0.00 & $\mathrm{i}$ & $<0.04:^{8}$ & - & $-\quad-$ & - & - & - & $\ldots$ & - & & - \\
\hline 7771.94 & O I (1) & 9.14 & $\mathrm{~s}$ & $0.22^{7} \mathrm{~b}$ & 370 & $1.03^{4} \mathrm{~b}$ & 270 & $1.38 \mathrm{~b}$ & 240 & $0.64 \mathrm{~b}$ & $\longrightarrow$ & & - \\
\hline 7774.17 & O I (1) & 9.14 & $\mathrm{~s}$ & $"$ & - & $"$ & - & $"$ & - & $"$ & - & & - \\
\hline 7775.39 & O I $\quad(1)$ & 9.14 & $\mathrm{~s}$ & $"$ & - & $"$ & - & $"$ & - & $"$ & 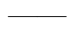 & & - \\
\hline 8437.96 & $\mathrm{P} 18$ & 12.04 & $\mathrm{p}$ & $0.07^{7} \mathrm{~b}$ & 403 & $0.50^{4} \mathrm{~b}$ & 622 & 0.10: & 185 & 0.33 & 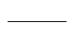 & & - \\
\hline 8446.25 & O I $\quad(4)$ & 9.48 & $\mathrm{~s}$ & $"$ & - & $"$ & - & $0.19 \mathrm{~b}$ & 110 & $0.17 \mathrm{~b}$ & 0.3 & $10 \mathrm{~b}$ & - \\
\hline 8446.36 & O I (4) & 9.48 & $\mathrm{~s}$ & $"$ & - & $"$ & - & $"$ & - & $"$ & $"$ & & - \\
\hline 8446.76 & O I (4) & 9.48 & $\mathrm{~s}$ & $"$ & - & $"$ & - & $"$ & - & $"$ & $"$ & & - \\
\hline 8498.02 & Ca II (2) & 1.69 & $\mathrm{~s}$ & $0.10^{7} \mathrm{~b}$ & 440 & $0.38^{4} \mathrm{~b}$ & 500 & -.90 & 150 & & 2.6 & $10 \mathrm{~b}$ & 124 \\
\hline 8502.49 & P16 & 12.04 & $\mathrm{p}$ & & - & & - & & - & & $"$ & & - \\
\hline 8542.09 & Ca II (2) & 1.69 & $\mathrm{~s}$ & $0.25^{7} \mathrm{~b}$ & 359 & $1.82^{4} \mathrm{~b}$ & 495 & 0.77 & 150 & & 3.9 & $10 \mathrm{~b}$ & 185 \\
\hline 8545.38 & $\mathrm{P} 15$ & 12.04 & $\mathrm{p}$ & & - & & - & & - & - & $"$ & & - \\
\hline 8598.39 & $\mathrm{P} 14$ & 12.04 & $\mathrm{p}$ & $0.05^{7}$ & 396: & 2.21 & 662 & - & - & - & 0.5 & ${ }^{10} \mathrm{~b}$ & 122 \\
\hline 8662.14 & Ca II (2) & 1.69 & $\mathrm{~s}$ & $0.26^{7} \mathrm{~b}$ & 393 & $4.27^{4} \mathrm{~b}$ & 700 & - & - & - & 2.4 & 101 & 212 \\
\hline 8665.02 & $\mathrm{P} 13$ & 12.04 & $\mathrm{p}$ & 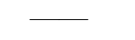 & - & 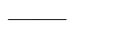 & - & - & - & - & $"$ & & - \\
\hline
\end{tabular}

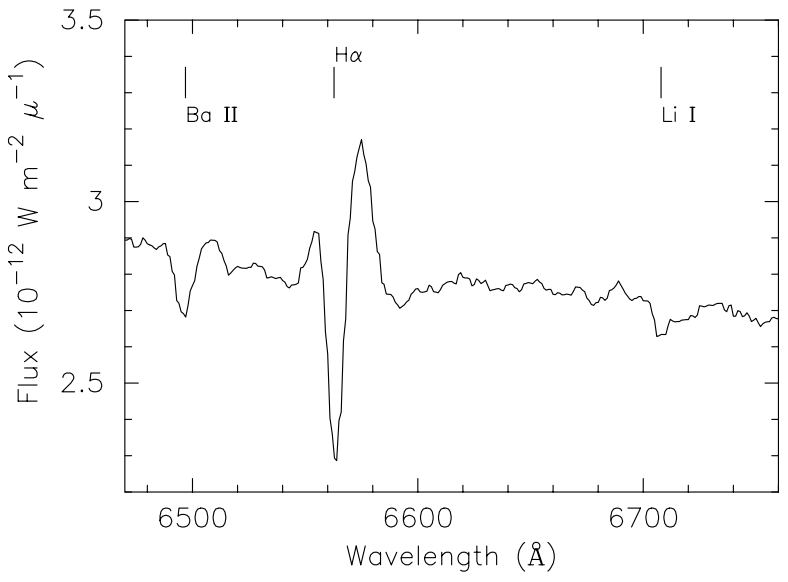

Fig. 3. Part of the low-dispersion blue spectrum of BN Ori, taken with the ESO $1.5 \mathrm{~m}$ telescope in Dec'94

star HR 5999 (Bessell \& Eggen 1972; Tjin A Djie et al. 1989; Baade \& Stahl 1989).

\subsubsection{Blue spectra}

Two low-dispersion blue spectra have been taken: one in 1983 at Mt. Maidanak and one in 1994 at ESO (Fig. 3). From the strength of the $\mathrm{H} \gamma, \mathrm{H} \delta, \mathrm{H} \varepsilon$ and $\mathrm{Ca}$ II $\mathrm{K}$ lines and the presence of the $G$-band in the spectra we confirm the classification F2 - F3 by Kolotilov \& Zajtseva (1976).
However, some additional lines, such as the strong Ca I $4227 \AA$ A line, may point to a later type, e.g. F3 - F5. These blue spectra again show many absorption lines, among which the Fe II multiplet 42 , the $G$-band, the Na I D doublet and the Ba II (6497 $\AA$ ) and Li I (6708 $\AA$ ) lines which are also conspicious lines in FUOR spectra (Reipurth 1990). The EW of the Li I line is comparable (Table 6) with that of $\mathrm{Li}$ I in the spectrum of FU Ori (Herbig 1965).

The red emission-component of $\mathrm{H} \alpha$ is stronger than the blue one, in contrast with the $\mathrm{H} \alpha$-profile in the lowdispersion IDS spectrum of 1986, which shows roughly equal red and blue emission-components.

\subsubsection{Red spectra}

Two low-dispersion spectra in the far-red wavelength range have been secured by us: one in 1983 at Mt. Maidanak and one in 1992 at ESO. Figure 4 shows part of the spectrum obtained at ESO and Table 6 lists the EWs and FWs of the most important lines in this spectrum and in those of FU Ori (Shanin 1979) and HR 5999 (Tjin A Djie et al. 1989). Figure 4 shows the Ca II (2) IRtriplet $(8498,8542$ and $8662 \AA)$ in absorption, which is blended with the Paschen absorption lines P16, P15 and P13, respectively. We have used the method of Bychkov et al. (1978) to make a spectral classification from the EWs of the blends of the CaII (2) IR-triplet and the EW of the unblended P14. In the assumption that none of 


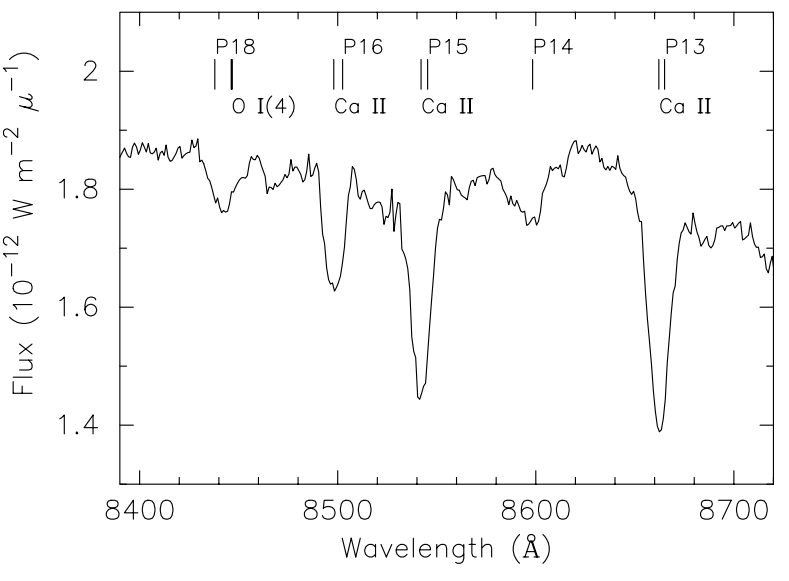

Fig. 4. Part of the low-dispersion red spectrum of BN Ori, taken with the ESO $1.5 \mathrm{~m}$ telescope in Dec'92, with the most prominent lines identified

these lines are filled-in by emission one can derive a lower limit for the effective temperature or the spectral type of the region of formation of these lines. From Table 6 we find for BN Ori an EW ratio of $\sim 12$, which points to a spectral type around G5. With the same method Shanin (1979) classified FU Ori as a K0 star. A reticon spectrum of HR 5999 (Tjin A Djie et al. 1989) in the same spectral region gives us an EW ratio of 3.1 , which indicates a spectral type around A5. High-resolution observations of only the 8498 and $8542 \AA$ lines (Hamann \& Persson 1992) show the unblended profiles with indications for some fillingin by emission. After correction for emission the EWs would be somewhat larger, which could move the spectral type of HR 5999 to a slightly later type, e.g. A7. Similar (unknown) emission corrections to the Ca II (2) IR-triplet for BN Ori and FU Ori could shift their classifications to types later than G5 and K0 respectively. In the far-red spectrum we also observed the two O I IR-triplets around 7774 and $8446 \AA$ (blended with $\mathrm{P} 18$ at $8438 \AA$ ). The EWs of these lines can be compared with those of standard stars of well-known spectral class (Slettebak 1986; Figs. 2 and 3, Faraggiana et al. 1988). High-resolution profiles of these lines for HR 5999 have been given by Felenbok et al. (1988) and by Hamann \& Persson (1992). The EW, measured from the best profile of the O I (1) IRtriplet is $1.38 \AA$, which according to Slettebak (1986; Fig. 2) is consistent with A2 - A7 IIIsh. A correction for the emission-component would not change this conclusion. The EW of this (unblended) triplet for BN Ori is $0.22 \AA$, which would classify the star as F6 - G0, unless the contribution by emission is very large. A comparison of the EW of the blended O I (4) IR-triplet for HR 5999 with Fig. 3 of Slettebak's paper gives again A2 - A7 IIIsh, even if the filling-in by emission (Hamann \& Persson 1992) is taken into consideration. The very small $\mathrm{EW}$ of this line for BN Ori suggests again a type G, but F0 could be possible if the correction for emission fill-in would raise the EW from 0.1 to $0.4 \AA$. For FU Ori the EW of this line is $0.3 \AA$ (Shanin 1979). This could lead to a classification F3 IV. Since FU Ori has strong shell features and high luminosity-class (see next section) its spectral type must be later than A6 - G0 (Slettebak 1986; Fig. 3), unless the emission contribution to the O I (4) IR-triplet is very strong.

\subsection{High-dispersion spectra}

A high-dispersion visual spectrum of BN Ori was obtained on Nov. 21, 1991 with the main stellar spectrograph (MSS) mounted on the $6 \mathrm{~m}$ Big Azimuthal Telescope (BTA) of the Special Astrophysical Observatory (SAO) in the Northern Kaukasus. Various parts of this spectrum are given in Fig. 5 and the values of the EWs and FWs of the stronger lines are listed in Table 11, together with those of HR 5999 determined from the Coudé spectrum G9359 $\left(\boldsymbol{D}=12.4 \AA \mathrm{mm}^{-1}\right.$, Tjin A Djie et al. 1989), and some qualitative information concerning line strengths from the Coudé spectra of FU Ori $\left(\boldsymbol{D}=16 \AA \mathrm{mm}^{-1}\right.$, Herbig 1966).

In this table the identification of the lines with wavelengths longer than $5200 \AA$ is tentative, except for the Ba II $(6497 \AA)$ and the Li I $(6708 \AA)$ lines. From the width of several photospheric lines (MgII (4), Fe II (42) and Fe I (43)) we estimate a rotation rate of $v \sin i=180-$ $230 \mathrm{~km} \mathrm{~s}^{-1}$ for BN Ori. A similar estimate for HR 5999 gives $v \sin i \sim 135 \mathrm{~km} \mathrm{~s}^{-1}$. However, a comparison with the Mg II (4481 $\AA)$ line-width with those of rotation standard stars (Slettebak et al. 1975), taken with the same instruments as used for HR 5999, leads to $v \sin i=180$ $\pm 20 \mathrm{~km} \mathrm{~s}^{-1}$ for this star (Tjin A Djie et al. 1989). This suggests that the rotation rate of BN Ori is probably somewhat higher, perhaps around $250 \mathrm{~km} \mathrm{~s}^{-1}$. For FU Ori Herbig (1966) derived $v \sin i \approx 60 \mathrm{~km} \mathrm{~s}^{-1}$.

The most important conclusion from the spectrum of BN Ori is that different lines suggest different spectral types for the star:

1. from a comparison of the EWs of the higher terms of the Balmer series with a statistical analysis made by Kopylov (1958) we estimate a spectral type A6 - A7 for stars with low and average luminosity,

2. the strength of many FeII and Ti II lines, usually known as photospheric ones is consistent with spectral types A6 - F0,

3. the strenght of the $G$-band near $4300 \AA$ points to a type F0 - F2 or early G,

4. the EWs of the rather numerous Fe I lines are typical for spectral types F0 to G5,

5. the strength of the MnI resonance triplet at $4033 \AA$ suggests a late $G$ - or early K-type sub-class.

Together with the classifications G0 from the OI (1) IR-triplet and G5 from the CaII (2) IR-triplet (Sect. 4.2.2) this variety in spectral type indicates a considerable thermal stratification of the atmosphere of 


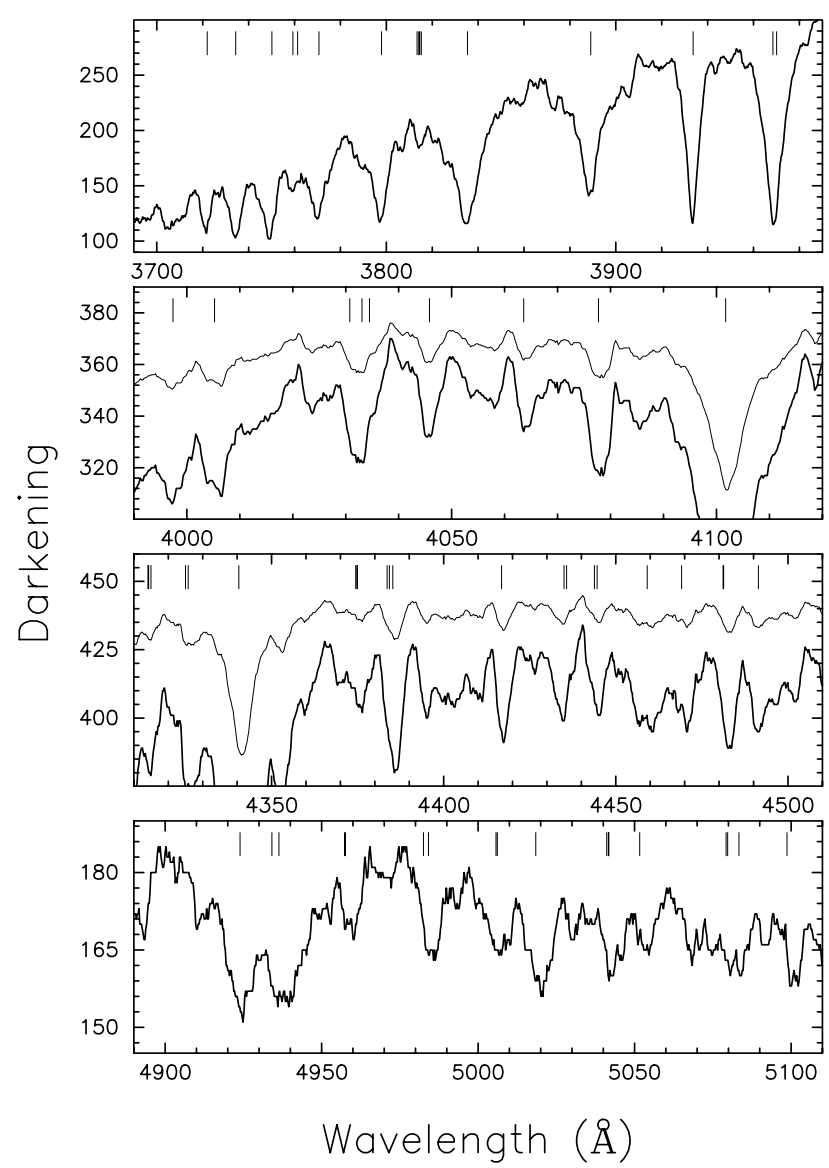

Fig. 5. Various parts of the high-dispersion visual spectrum of BN Ori obtained at SAO in 1991. Shown are from top to bottom: • CaII $\mathrm{H}$ and $\mathrm{K}, \mathrm{H} \varepsilon$ to $\mathrm{H} 14$ and the TiII lines at $3760 \AA, \bullet \mathrm{H} \delta$ and its blue neighbourhood (V II, Sr II, Fe I, Ti II, Mn I (2) triplet), $\bullet \mathrm{H} \gamma$ and the red neighbourhood, $\bullet$ the red neighbourhood of $\mathrm{H} \beta$. The identification of the marked lines can be found in Table 11

BN Ori, which must consist of an A6 - A7 photosphere, surrounded by a rather dense and cooler envelope or disc. Several lines such as Sr II (4078 $)$ and the semi-forbidden TiII (13) lines near $3760 \AA$ are typical shell lines and are probably formed in the low-density outer part of the envelope.

Most of the lines mentioned above are also present in the spectra of FU Ori and HR 5999 which were obtained with comparable resolution. The visual spectrum of FU Ori has been classified as F2p:I - IIe (Herbig 1966). If we add the classification F6 - G0 from the O I (4) IR-triplet and K0 from the Ca II (2) IR-triplet we can conclude that also the atmosphere of FU Ori is thermally stratified. Similar to BN Ori the spectrum of FU Ori shows a strong $G$-band and strong photospheric lines of Ba II and LiI. However, in contrast with BN Ori, strong components with velocities around $-50 \mathrm{~km} \mathrm{~s}^{-1}$ are present in the spectrum of FU Ori for all lines of Hydrogen and neutral or singly-ionised metals (with exception of the Fe I lines with higher excitation levels). Their presence has led to the qualification peculiar in the spectral classificaton. Because of their difference in velocity with the photospheric lines these components are probably formed in an outer shell region. Since we have no EWs from the visual spectrum of FU Ori, with the exception of Li I, Na I D and $\mathrm{H} \alpha$ (Table 6 ), we only give qualitative estimates from Herbig (1966). With exception of the emission in CaII K and $\mathrm{H} \alpha$, the spectrum did not seem to vary significantly in time between 1945 and 1963.

The visual spectrum of HR 5999 has been classified as A5 - A7 IIIe (Bessell \& Eggen 1972; Tjin A Djie et al. 1989). The classification from the farred spectrum (Sect. 4.2.2) is again A7 III, which indicates an absense of significant thermal stratification of the envelope of HR 5999. This means that the stratification of the atmosphere as found in BN Ori and FU Ori can not originate from the disc component, because this component is significant in the case of HR 5999 (Sect. 5). We will discuss this further in Sect. 6 .

In contrast with FU Ori and BN Ori we do not find a $G$-band and Li I line in the spectrum of HR 5999, but similar to FU Ori, it has strong blue-shifted ( -30 to $-50 \mathrm{~km} \mathrm{~s}^{-1}$ ) components of the Balmer- and metalliclines, which are formed in a cool, dense outer shell (Tjin A Djie et al. 1989). Such an dense cool circumstellar shell does not seem to be present around BN Ori.

This is confirmed by the high-resolution observations of the NaID (Fig. 6) with the CAT at $\operatorname{ESO}(\boldsymbol{R}=$ $\left.0.11 \AA \mathrm{pix}^{-1}\right)$ and the MSS at $\mathrm{SAO}\left(\boldsymbol{R}=0.65 \AA \mathrm{pix}^{-1}\right)$ and the K I lines (not shown). The observations at ESO show the presence of interstellar components only, with a heliocentric-velocity of $+26 \mathrm{~km} \mathrm{~s}^{-1}$, which is of the same order as those of other stars in the Orion complex. The interstellar Na I D lines have EWs equal to $0.13 \AA\left(D_{2}\right)$ and $0.09 \AA\left(D_{1}\right)$. Since these lines are not saturated, we can use the Strömgren (1948) method, with which we derive an interstellar (foreground) column density for Na I of $1.2710^{12} \mathrm{~cm}^{-2}$. With the relation between $E(B-V)$ and NaID column density of Hobbs (1974) we find for the foreground of BN Ori an $E(B-V)$ of 0 . 07 . From the Na ID lines, observed at SAO in 1991, we obtain the same value. These values are in agreement with those determined for the Orion complex by Walker (1969). In contrast with the NaID lines of BN Ori, those of HR 5999 and FU Ori have several velocity-components which are formed in the circumstellar envelope of the star. The velocities measured for HR 5999 are $v_{\max } \sim-35 \mathrm{~km} \mathrm{~s}^{-1}$, and for FU Ori $v_{\max } \sim-100 \mathrm{~km} \mathrm{~s}^{-1}$ (Bastian \& Mundt 1985; Reipurth 1990). The circumstellar origin of the Na I D lines in the spectrum of HR 5999 is clear from the variability of their EWs and the absence of NaID lines in the spectrum of the common proper motion companion HR 6000 (Tjin A Djie et al. 1989). 


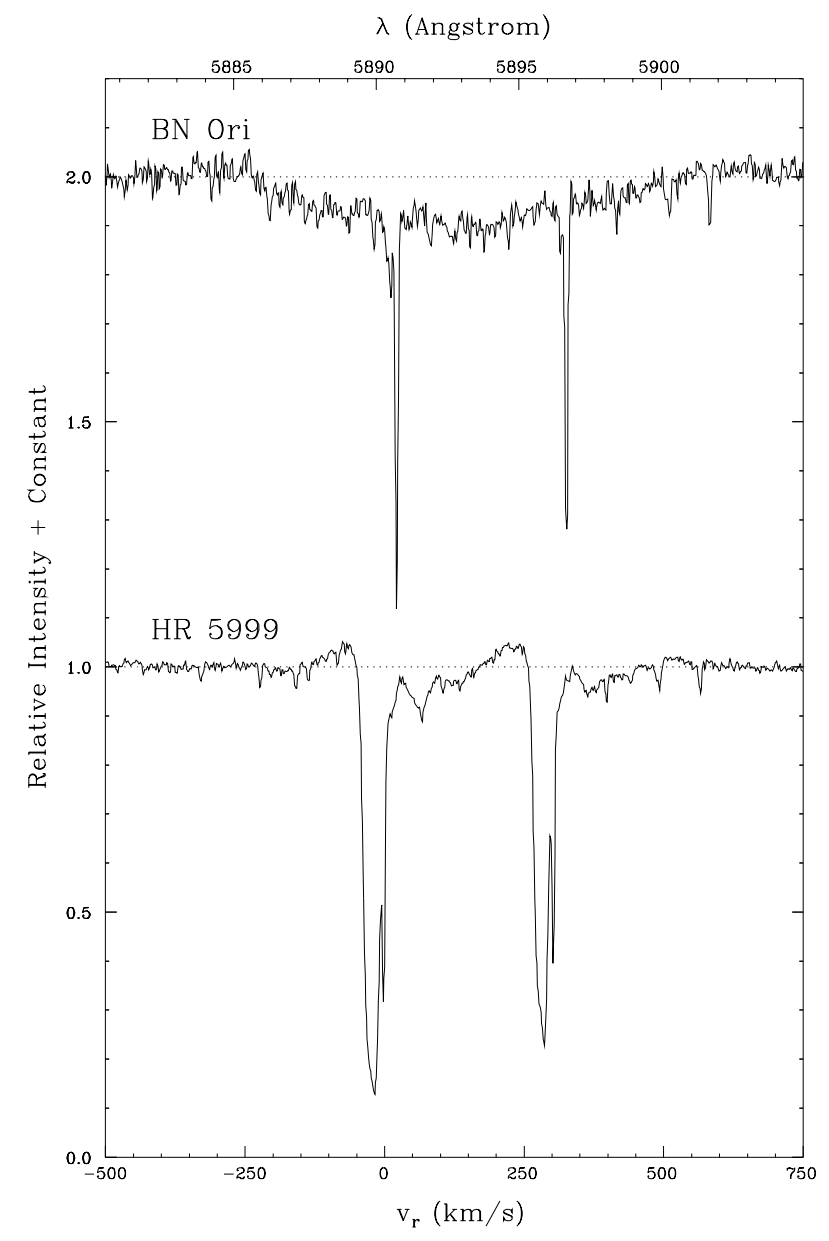

Fig. 6. High-resolution heliocentric-corrected Na I D spectra obtained at ESO in 1994 for BN Ori (top) and HR 5999 (bottom, see also Grady et al. 1996). The dotted lines indicate the continuum level. The spectrum of HR 5999 was used for the annotation and the difference in radial velocity between the two galactic directions of observation introduces a wavelength-shift in the figure for the spectrum of BN Ori

The KI resonance lines of BN Ori observed at ESO (Jan. 20, 1995) were very weak. The $7699 \AA$ line is hardly distinguishable from the noise, while the $7665 \AA$ line is in the wing of a rotational line of the telluric $\mathrm{A}$ band of $\mathrm{O}_{2}$. The lines have a heliocentric-velocity of $\sim+20 \mathrm{~km} \mathrm{~s}^{-1}$ and are of interstellar origin. The EWs of these lines are $8.5 \mathrm{~m} \AA( \pm 30 \%)$ and $\leq 4 \mathrm{~m} \AA( \pm 50 \%)$ for the 7665 and $7699 \AA$ line, respectively. If the $7665 \AA$ line is optically thin we derive with the oscillator strength of the line, a column density of $2.310^{10} \mathrm{~cm}^{-2}$. The ratio of the column densities of $\mathrm{Na}$ I to $\mathrm{KI}$ in the direction of BN Ori is then 54, the same value as for the direction of $\mathrm{AB}$ Aur (Felenbok et al. 1983).

A peculiar feature of BN Ori is the presence of broad, shallow and symmetric components of the NaID lines, with a FW of about $400 \mathrm{~km} \mathrm{~s}^{-1}$. The corresponding $v \sin i$ is close to the rotation rate $\left(\sim 220 \mathrm{~km} \mathrm{~s}^{-1}\right)$ of the photo- sphere of BN Ori, which has $T_{\text {eff }}=7150 \mathrm{~K}$. Because of the low ionization potential of $\mathrm{Na}$ I $\left(E_{\chi}=5.14 \mathrm{eV}\right)$, we do not expect to observe these lines from photospheres with $T_{\text {eff }}>5500 \mathrm{~K}$. However, the broad components can originate from the atmosphere of the inner part of the accretion-disc, which has a temperature below $5890 \mathrm{~K}$ (Table 7) and a $v \sin i$ which is close to the photosphericrotation rate. A similar broad component can also be found in the high-resolution profiles of the Na I D lines of HR 5999. However, in this case its width and EW are more difficult to estimate because of the circumstellar emissioncomponent in both NaI D lines. The values for HR 5999 in Table 6 are therefore probably lower limits. The large width of the circumstellar components in the NaI D profiles of FU Ori (Reipurth 1990) does not enable us to detect the presence of such shallow components for this star.

The $\mathrm{H} \alpha$-profile of BN Ori has been observed by us at three dates in high-resolution: in Nov'91 at the SAO, and twice in Jan'95 at ESO. An additional profile obtained on Nov. 10, 1989 by Fernández et al. (1995) is very similar to the one of Nov'91. The resulting profiles are also similar to those given by Kolotilov \& Zajtseva (1976), but show more details. In order to estimate the chromospheric profile we divided the normalised fluxes in the observed profiles by the corresponding normalised fluxes of the profiles of $21 \mathrm{Vul}\left(\mathrm{A} 7-\mathrm{F} 0 \mathrm{IV}, v \sin i \sim 200 \mathrm{~km} \mathrm{~s}^{-1}\right.$ ), which has a very weak shell. The profile of $21 \mathrm{Vul}$ was taken from Doazan et al. (1991) and a contribution from the shell to $\mathrm{H} \alpha$ is only expected in the minimum of the profile. After this procedure, the $S A O \mathrm{H} \alpha$-profile shows a single emission peak with a single, broad absorptionfeature with $v \sim-650 \mathrm{~km} \mathrm{~s}^{-1}$, which agrees well with the velocities found by Zajtseva \& Kolotilov (1973). One of the ESO $\mathrm{H} \alpha$-profiles has an almost symmetrical shape, with a relative central part which is almost equal to that of the $S A O \mathrm{H} \alpha$-profile. The other profile is less strong in its central part and has a depression near $+240 \mathrm{~km} \mathrm{~s}^{-1}$. The three profiles and their "chromospheric" shapes are shown in Fig. 7. The shapes are different from those of HR 5999 (Tjin A Djie et al. 1989; Baade \& Stahl 1989; Praderie et al. 1991), which show no high-velocity absorption-features. The $\mathrm{H} \alpha$-emission flux of BN Ori can be derived from Fig. 7 by integration and normalisation with the continuum flux near $6600 \AA$, obtained from the SED with the help of the distance to radius ratio and after correction for extinction (Sects. 2 and 4.3). In this way we find emission fluxes of $1.5610^{5} \mathrm{~W} \mathrm{~m}^{-2}$ for the observations of Nov. 10, 1989 and Nov. 21, 1991, and $1.2210^{5} \mathrm{~W} \mathrm{~m}^{-2}$ for the observations of Jan. 20, and Jan. 17, 1995. In the same way we may derive the $\mathrm{H} \alpha$ emission flux of $\mathrm{HR} 5999$ in its phase of maximum brightness $V^{\mathrm{J}}=6{ }^{\mathrm{m}} 8$ (Tjin A Djie et al. 1989), which after an estimated correction for the central absorption, results to $2.3-3.410^{6} \mathrm{~W} \mathrm{~m}^{-2}$. For $V^{\mathrm{J}}=7^{\mathrm{m}} \cdot 6$ it is $5.810^{6} \mathrm{~W} \mathrm{~m}^{-2}$. The flux of HR 5999 is therefore larger than that of BN Ori by a factor which is in the range $22-28$. 


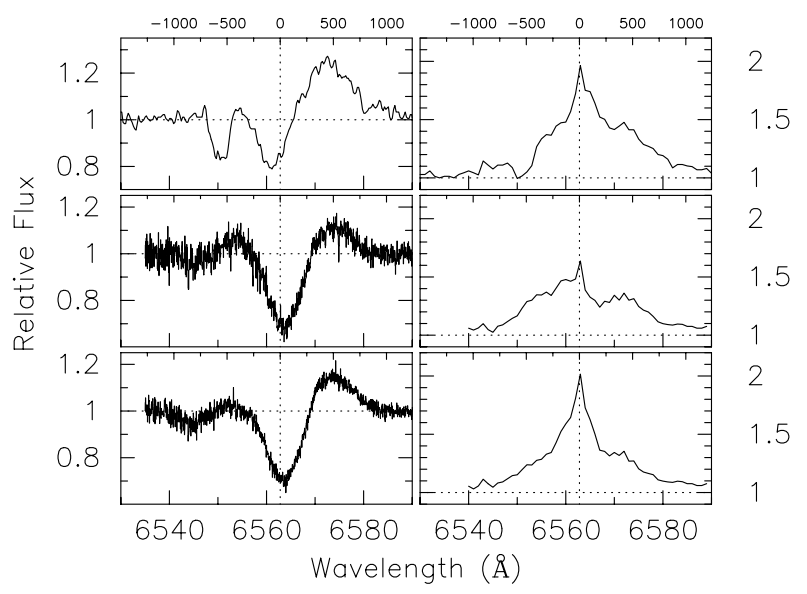

Fig. 7. Left: high-resolution $\mathrm{H} \alpha$-profiles of BN Ori obtained at SAO in 1991 (top) and at ESO on Jan. 171995 (middle) and Jan. 201995 (bottom). Right: corresponding profiles after re-binning and division by the normalised $\mathrm{H} \alpha$-profile of $21 \mathrm{Vul}$ (from Doazan et al. 1991). Top-axis gives the velocity $\left(\mathrm{km} \mathrm{s}^{-1}\right.$ ) relative to $6562.82 \AA$

The H $\alpha$-profiles of FU Ori (Bastian \& Mundt 1985; Reipurth 1990) have P-Cygni shapes, with a very broad and deep absorption trough. Outflow velocities as high as $-350 \mathrm{~km} \mathrm{~s}^{-1}$ can be estimated from these profiles. The observed profile has been corrected for photospheric contributions with the use of calculated $\mathrm{H} \alpha$-profiles (Kurucz $1979)$ of a F0 - F2 Ib photosphere $\left(T_{\text {eff }}=7500 \mathrm{~K}\right.$, $\log g=2.0)$. The extinction-corrected surface-flux of the symmetrised $\mathrm{H} \alpha$-profile is then $3.810^{5} \mathrm{~W} \mathrm{~m}^{-2}$. The $\mathrm{H} \alpha$ emission fluxes are collected in Table 9.

\section{UV-spectroscopy}

Several low-resolution UV-spectra of BN Ori have been obtained with the International Ultraviolet Explorer (IUE) satellite in 1984 and 1986. Details of the observations are given in Table 5 . Before each exposure the brightness of BN Ori was measured by the Fine Error Sensor (FES) of IUE in the Field Camera mode (Barylak 1989). In all cases the resulting $V^{\mathrm{J}}$ magnitude was around 9.68 , which is close to the results of the ground-based photometry in the periods around the IUE observations (Tables 3 \& 12). The low-resolution UV-spectra of BN Ori, HR 5999 and several other Herbig Ae stars show strong emission lines of O I, C II, Si IV and CIV (Tjin A Djie et al. 1982; Brown et al. 1986) shortward $1600 \AA$. The high intrinsic radiation flux in these lines can probably only be supplied by accretion (Tjin A Djie \& Blondel 1997) and it has been possible to derive information on the accretion parameters of $\mathrm{A}$ and $\mathrm{F}$ stars from the UV-continuum over the full wavelength range $(1150-3200 \AA)$ of IUE (Blondel \& Tjin A Djie 1997). After the choice of a stellar spectral type and luminosity-class and addition of the observed visual magnitude, the distance and the fore- ground colour excess, we have matched the observed UVcontinuum with standard star spectra from the IUE spectral atlasses for the photospheric and boundary-layer components and a optically-thick model for the accretion-disc (UV3C, Blondel \& Tjin A Djie 1994, 1997).

The results of the parameter adjustments required for the matching of the low-resolution UV-spectra of BN Ori, HR 5999, BF Ori and FU Ori are collected in Table 7. The masses of the stars $\left(M_{\star}\right)$ were estimated from the stellar radii ( $R_{\star}$ derived from the model calculation) and the photospheric $T_{\text {eff }}$ (Schmidt-Kaler 1982) with the use of the evolutionary tracks of Palla \& Stahler (1993). With this mass and radius, the width of the boundary layer $\left(\delta_{\mathrm{bl}}\right)$ and its effective temperature, the corresponding massaccretion rate $(\dot{M})$ was derived. Since the mass of FU Ori cannot be derived from the evolutionary tracks, we have assumed that its precursor was a $1.0 M_{\odot}$ T Tauri star. In general the stellar spectral type and luminosity-class appear to be strongly constrained (to 1 or 2 subtypes) by the observed UV-continuum. For BN Ori we estimated a distance of $\sim 400 \mathrm{pc}$ from the data of Artyukhina (1959) and a foreground $E(B-V)=0.07$ from the Na I D interstellar components.

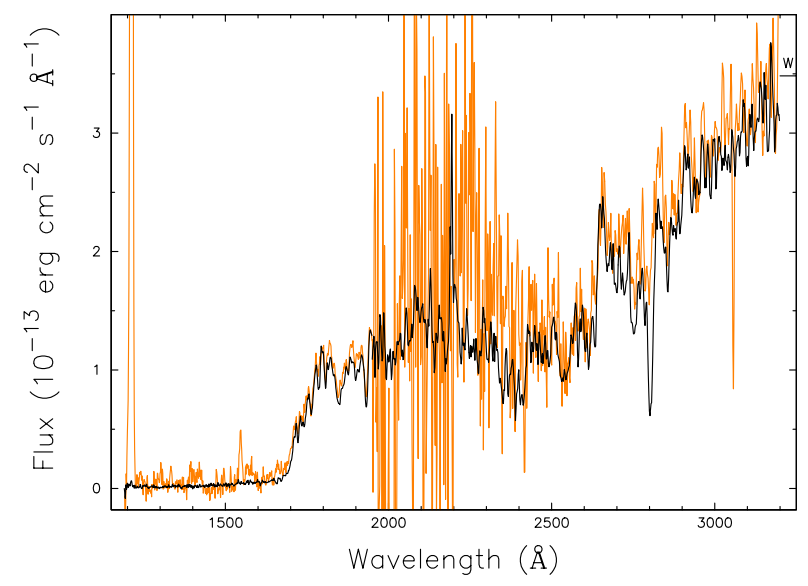

Fig. 8. Low-resolution UV-spectrum of BN Ori after correction for the foreground extinction (grey line) with the accretion model calculation (black line). The used model parameters are listed in Table 7, and the "W" indicates the Walraven $W$-band flux (Table 2) after correction for the foreground extinction. The low $\mathrm{S} / \mathrm{N}$ in the observed spectrum $(2000 \AA-2400 \AA)$ is due to the low sensitivity of the LWP camera in this range

The calculated spectrum and the SED corresponding to the parameters of BN Ori in Table 7 are given in Figs. 8 and 9. These results show that the UV3C-model gives a good agreement with the observations from the far-UV up to the $L$-band in the NIR. In general the model (with an optically thick disc up to $40 R_{\star}$ ) gives higher values than observed for the fluxes in the $K$ - and $L$-band. The disagreement could not be reduced by taking the disc 
Table 7. Parameters used in the UV3C-model calculations with the low-resolution UV-spectra. For the B9 Iab component we used $T_{\text {eff }}=10300 \mathrm{~K}$ (Schmidt-Kaler 1982). The value given by $\boldsymbol{E}^{1 \mathrm{~s}}$ is the interstellar (foreground) $E(B-V)$, while the $\boldsymbol{E}^{\text {tot }}$ value is the total excess

\begin{tabular}{|c|c|c|c|c|c|c|c|c|c|c|c|c|c|}
\hline \multirow[t]{2}{*}{ star } & \multirow{2}{*}{$\begin{array}{l}\text { Spec. } \\
\text { Type }\end{array}$} & $M_{v}$ & $V_{\text {lue }}^{\text {fes }}$ & $E^{1 \mathrm{~s}}$ & \multirow{2}{*}{$\begin{array}{c}\mathrm{d} \\
(\mathrm{pc})\end{array}$} & \multirow{2}{*}{$\begin{array}{c}R_{\star} \\
\left(R_{\odot}\right) \\
\end{array}$} & \multirow{2}{*}{$\begin{array}{c}L_{\star} \\
\left(L_{\odot}\right) \\
\end{array}$} & \multirow{2}{*}{$\begin{array}{c}M_{\star} \\
\left(M_{\odot}\right) \\
\end{array}$} & \multirow{2}{*}{$\begin{array}{r}v \sin i \\
\left(\mathrm{~km} \mathrm{~s}^{-1}\right)\end{array}$} & \multirow{2}{*}{$\begin{array}{l}\text { age } \\
\text { (Myr) }\end{array}$} & \multirow{2}{*}{\multicolumn{3}{|c|}{ Other Designations }} \\
\hline & & \multicolumn{3}{|c|}{ (magnitudes) } & & & & & & & & & \\
\hline BN Ori & F0 III & +1.30 & 9.67 & 0.07 & 390 & 3.04 & 21.9 & 2.1 & 220 & 4. & \multicolumn{2}{|c|}{ HD 245465} & $\mathrm{BD}+06^{\circ} 971$ \\
\hline HR 5999 & A5 III & -0.40 & 6.71 & 0.03 & 230 & 5.26 & 107. & 3.5 & 180 & 0.3 & \multicolumn{2}{|c|}{ HD 144668} & $\mathrm{CD}-38^{\circ} 10893$ \\
\hline BF Ori & A7 III & +1.40 & 9.90 & 0.06 & 415 & 2.54 & 20.0 & 2.0 & 100 & 6. & & & $\mathrm{BD}-06^{\circ} 1259$ \\
\hline FU Ori & F0 I & -2.00 & 9.30 & 0.06 & 500 & 11.8 & 441. & 1.0 & 60 & - & & & \\
\hline & & \multicolumn{2}{|c|}{ boundary layer } & \multicolumn{2}{|c|}{ disc } & & & & \multicolumn{3}{|c|}{ Relative $F_{v}$} & \multirow{2}{*}{\multicolumn{2}{|c|}{$\stackrel{\dot{M}}{\left(10^{-8} M_{\odot} \mathrm{yr}^{-1}\right)}$}} \\
\hline \multicolumn{2}{|c|}{ star } & $\begin{array}{l}\text { Spec. } \\
\text { Type }\end{array}$ & $\begin{array}{c}\delta_{\mathrm{bl}} \\
\left(R_{\star}\right)\end{array}$ & $\begin{array}{c}T_{\max }^{\mathrm{d}} \\
(\mathrm{K})\end{array}$ & $\begin{array}{l}i \\
\left(^{\circ}\right)\end{array}$ & $\begin{array}{c}A_{v}^{\mathrm{cs}} \\
(\mathrm{mag})\end{array}$ & $\begin{array}{l}R_{v}^{\mathrm{cs}} \\
-\end{array}$ & $\begin{array}{l}E^{\text {tot }} \\
(\mathrm{mag})\end{array}$ & $\begin{array}{l}\text { star } \\
(\%)\end{array}$ & $\begin{array}{l}\text { b.l. } \\
(\%)\end{array}$ & $\begin{array}{l}\text { disc } \\
(\%)\end{array}$ & & \\
\hline & Ori & B9 Iab & 0.002 & 5887 & 80 & 0.231 & 4.50 & 0.12 & 96.67 & 0.18 & 3.15 & 2.0 & \\
\hline & 5999 & B9 Iab & 0.005 & 6580 & 53 & 0.489 & 3.10 & 0.19 & 86.64 & 0.64 & 12.72 & 54.0 & \\
\hline & Ori & B9 Iab & 0.040 & 5810 & 60 & 0.190 & 3.10 & 0.12 & 86.00 & 5.00 & 9.00 & 32.0 & \\
\hline \multirow{2}{*}{\multicolumn{2}{|c|}{ FU Ori }} & B9 Iab & 0.020 & 6034 & 45 & 2.79 & 3.40 & 0.88 & 85.30 & 3.40 & 11.30 & 2300 & \\
\hline & & & & & 70 & 2.71 & 3.40 & 0.86 & 91.34 & 2.20 & 6.46 & & \\
\hline
\end{tabular}

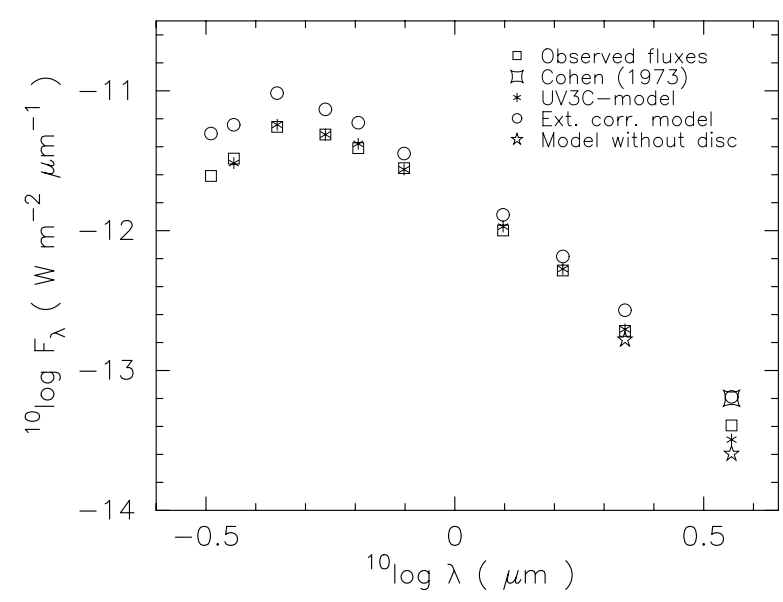

Fig. 9. SED of BN Ori. The fluxes derived from the photometry of Sect. 2 are indicated by squares. The $(*)$ points correspond to the fluxes calculated with the UV3C-model (Table 7), obtained by matching the low-resolution UV-spectrum (Fig. 8). The $K$ - and $L$-band fluxes (open star) show the effect of removing the disc contribution in the model-calculation. Circles indicate the extiction-corrected model results

optically thin but when we limit the outer radius of the disc to $2.0-2.5 R_{\star}$ the NIRE almost disappears (Fig. 9). This suggest that the disc has been largely dissipated during the outburst of 1947 . For the parameters in Table 7 the $E(B-V)$ is 0 . 12 , which with an observed $(B-V) \approx$ 0.45 corresponds with $(B-V)_{0}=0 .{ }^{\mathrm{m}} 33$. This value is slightly higher then the $0 .{ }^{\mathrm{m}} 30$ for spectral type $\mathrm{F} 0$ III given by Schmidt-Kaler (1982) but not unacceptable as BN Ori may be not exactly F0 III. Both the small circumstellar colour excess $(0.05)$ and the negligeable NIRE indicate that BN Ori has almost no circumstellar dust left.
Table 8. Identification, EW $(\AA)$ and $\mathrm{FW}\left(\mathrm{km} \mathrm{s}^{-1}\right)$ of lines in the high-resolution UV-spectra of BN Ori, HR 5999 and FU Ori. See Table 10 for the used symbols

\begin{tabular}{|c|c|c|c|c|c|c|c|c|c|c|}
\hline \multirow{2}{*}{$\begin{array}{l}\lambda_{0} \\
(\AA)\end{array}$} & \multirow{2}{*}{\multicolumn{2}{|c|}{ Line id. }} & \multicolumn{2}{|l|}{$E_{\chi}$} & \multicolumn{2}{|c|}{ BN Ori } & \multicolumn{2}{|c|}{ HR 5999} & \multicolumn{2}{|c|}{ FU Ori } \\
\hline & & & $(\mathrm{eV})$ & $S$ & EW & FW & EW & FW & EW & FW \\
\hline 2562.54 & Fe II & (64) & 0.98 & $\mathrm{~s}$ & 0.16 & 40 & 1.31 & 140 & 0.26 & 56 \\
\hline 2576.11 & Mn II & (1) & 0.00 & is & 0.22 & 46 & 0.98 & 135 & - & - \\
\hline 2585.88 & Fe II & ( 1 ) & 0.00 & is & 0.17 & 39 & 1.12 & 151 & 0.11 & 37 \\
\hline 2591.54 & Fe II & (64) & 1.04 & $\mathrm{~s}$ & 0.15 & 46 & 0.97 & 207 & 0.21 & 25 \\
\hline 2598.37 & Fe II & ( 1 ) & 0.05 & is & 0.16 & 26 & 1.31 & 123 & - & - \\
\hline 2599.40 & Fe II & ( 1$)$ & 0.00 & $\mathrm{~s}$ & 0.22 & 46 & 1.18 & 138 & 0.31 & 61 \\
\hline 2617.62 & Fe II & (1) & 0.08 & $\mathrm{~s}$ & 0.07 & - & 1.04 & 151 & - & - \\
\hline 2625.66 & Fe II & ( 1 ) & 0.05 & $\mathrm{~s}$ & 0.13 & 39 & 1.09 & 192 & 0.21 & 43 \\
\hline 2677.16 & Cr II & ( 8 ) & 1.53 & is & 0.09 & - & 0.80 & 108 & - & - \\
\hline 2684.54 & Mn II & (63) & 4.14 & $\mathrm{~s}$ & 0.10 & 51 & 0.46 & 85 & 0.36 & 48 \\
\hline 2692.83 & Fe II & (62) & 0.98 & $\mathrm{~s}$ & 0.11 & 44 & 0.78 & 94 & - & - \\
\hline 2724.88 & Fe II & (62) & 1.04 & $\mathrm{~s}$ & 0.10 & - & 0.79 & 84 & 0.24 & 45 \\
\hline 2727.54 & Fe II & (63) & 1.04 & $\mathrm{~s}$ & 0.09 & 40 & 0.96 & 110 & 0.28 & 38 \\
\hline 2743.20 & Fe II & (62) & 1.09 & $\mathrm{~s}$ & 0.12 & 43 & 1.05 & 175 & - & - \\
\hline 2746.49 & Fe II & (62) & 1.07 & $\mathrm{~s}$ & 0.14 & - & 0.78 & 186 & 0.48 & 23 \\
\hline 2755.73 & Fe II & (62) & 0.98 & $\mathrm{~s}$ & 0.11 & - & 1.06 & 141 & 0.17 & 29 \\
\hline 2757.72 & Cr II & ( 6 ) & 1.50 & $\mathrm{~s}$ & 0.11 & 43 & 0.59 & 122 & - & - \\
\hline 2795.52 & Mg II & ( 1 ) & 0.00 & is & - & - & - & - & - & - \\
\hline 2802.70 & Mg II & ( 1 ) & 0.00 & is & - & - & - & - & - & - \\
\hline 2843.24 & Cr II & (5) & 1.52 & $\mathrm{~s}$ & 0.08: & 33 & 0.96 & 93 & 0.20 : & 28: \\
\hline 2849.83 & Cr II & ( 5$)$ & 1.50 & $\mathrm{~s}$ & 0.09: & 36 & 0.97 & 105 & 0.20 & 56 \\
\hline \multirow{2}{*}{\multicolumn{2}{|c|}{$2852.12 \mathrm{Mg}$ I }} & ( 1$)$ & 0.00 & $\mathrm{~s}$ & 0.09 & 14 & 1.05 & 124 & 0.26 & 56 \\
\hline & & & & $\mathrm{i}$ & 0.00 & - & - & - & 0.27 & 50 \\
\hline
\end{tabular}

In addition to the continua of the UV-spectra of BN Ori, we have also information on the individual lines from the high-resolution LWP image obtained with IUE. In spite of its long exposure $\left(13^{\mathrm{h}}\right)$ the $\mathrm{S} / \mathrm{N}$ ratio is very low in the less sensitive part of the LWP camera (shortward $2400 \AA$ ) but the region longward $2560 \AA$ is better exposed. Since the three low resolution UV-spectra of BN Ori show no differences (although they were taken at 
different times) we assume that the high-resolution spectrum of Nov.13, 1986 is representative for at least the period Jan'84 - Nov'86. The FES-magnitude $\left(V_{\text {lue }}^{\text {fes }}\right)$ during this observation agrees with a simultaneous ground-based photometric observation from DASA. From the comparison of the high-resolution UV-spectra of BN Ori, FU Ori and HR 5999 we can draw the following conclusions:

1. The presence of an extended cool shell around the three stars is revealed by the many low-excitation absorption lines of FeII, CrII and MnII in their UVspectra longward $2300 \AA$. The lines are strongest in the spectra of HR 5999 and the correlation of their EWvariations with the variations in brightness of HR 5999 (Blondel et al. 1989) shows that these lines are formed in the shell of the star. Because of the lower brightness of $\mathrm{BN}$ Ori and $\mathrm{FU}$ Ori the $\mathrm{S} / \mathrm{N}$ ratio in their spectra is lower than in the spectrum of HR 5999. In spite of this we can give upper limits for the EWs of the lines in the BN Ori spectrum and rough estimates $( \pm 30 \%)$ of the EWs of the lines in the FU Ori spectrum. Table 8, in which the EWs and FWs of the three stars are listed, shows that the EWs and FWs of the BN Ori lines are smaller than those of the corresponding lines in the spectrum of FU Ori, and both are smaller than the EWs and FWs of HR 5999. Since the spectral types of the three stars are not too different we expect that the column densities in the shells will also decrease from HR 5999 through smaller values for FU Ori to almost negligible values for BN Ori.

2. The Mg I resonance line $(2852.120 \AA)$ of BN Ori has two components: an interstellar one (with $\mathrm{EW}=$ $0.21 \AA$ ) which is displaced by $+26.5 \mathrm{~km} \mathrm{~s}^{-1}$ from its heliocentric position (determined from the Na I D interstellar lines) and a circumstellar one (with EW = $0.09 \AA$ ) with a velocity of $-53 \mathrm{~km} \mathrm{~s}^{-1}$ with respect to the interstellar one (Fig. 11). Similar to the NaID lines the continuum in the neighbourhood suggests that there exists a third, broad $\left(\mathrm{FW}=\sim 400 \mathrm{~km} \mathrm{~s}^{-1}\right)$ and shallow component, centered at the location of the two narrow components. Because of its width this component could be formed either at the stellar photosphere or near the inner accretion-disc. The Mg I line of FU Ori also appears to be double. Both components have an EW of about $0.26 \AA$ and the circumstellar component has a velocity of $\sim-67 \mathrm{~km} \mathrm{~s}^{-1}$ with respect to the interstellar component. The EW of the circumstellar component is larger than the corresponding one for $\mathrm{BN}$ Ori, while the foreground interstellar components of FU Ori and BN Ori are of comparable strength. The Mg I components in the UV-spectra of HR 5999 are not resolved because of the large width of the circumstellar component. Its EW is about 10 times larger than the upper limit of the EW of the corresponding component for BN Ori and $3-4$ times the corresponding EW for FU Ori.
3. The MgIIh (2802.7 $\AA$ ) and $\operatorname{Mg} I \mathrm{k}(2795.5 \AA)$ lines in the high-resolution spectrum of BN Ori have broad absorption-troughs with weak emission-components inside (Fig. 10). An accurate determination of the emission-components is difficult because of the presence of a reseau mark at $2795 \AA$ and an echelle order overlap between $2798 \AA$ and $2801 \AA$. Nevertheless we have made an attempt to derive some information on the shell component by dividing the (normalised) observed fluxes in the MgII profiles of BN Ori by the corresponding ones in the profiles of $21 \mathrm{Vul}$, obtained from the IUE archives (LWR 9037). This star has a spectral type A7 - F0 IV, a $v \sin i$ of $\sim 200 \mathrm{~km} \mathrm{~s}^{-1}$ and a very weak shell component in the Mg II profiles.

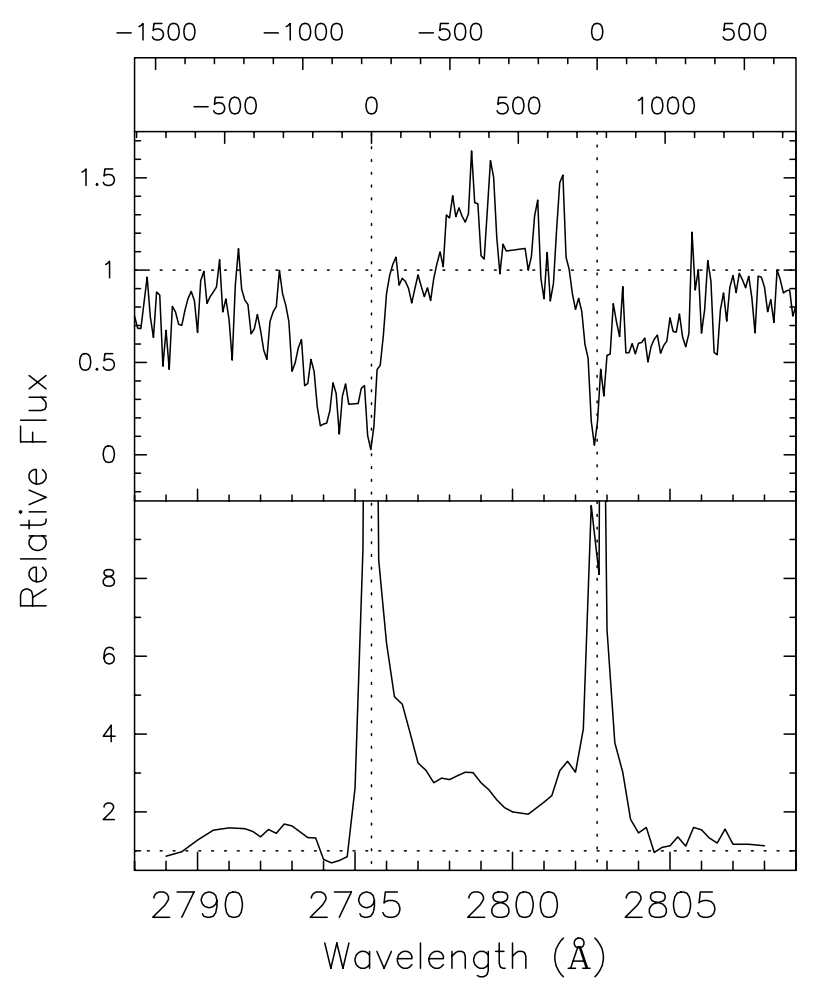

Fig. 10. Observed Mg II h\&k profiles of BN Ori (LWP 9512) and the residual profiles after normalisation to the $21 \mathrm{Vul}$ (LWR 9037) profiles. Top-axis give the velocity $\left(\mathrm{km} \mathrm{s}^{-1}\right)$ relative to the wavelengths of the $\mathrm{Mg}$ II h\&k lines

The result is given in Fig. 10, which shows that the shell component of $\mathrm{Mg}$ II has a P-Cygni profile with outflow-velocities up to $-250 \mathrm{~km} \mathrm{~s}^{-1}$. We have made a rough estimate of the $\mathrm{Mg} I \mathrm{k}$ emission-component $(\mathrm{EW} \approx 15 \AA)$ by assuming that the intrinsic emission (without the blue absorption trough) is symmetric. With the continuum flux near $2790 \AA$ from the low-resolution UV-spectra of BN Ori 
(2.5 $\left.10^{-13} \mathrm{erg} \mathrm{cm}^{-2} \mathrm{~s}^{-1}\right)$, the distance to radius ratio from the UV3C-model $\left(4.3510^{9}\right)$ and the total extinction correction (a flux factor of 2.08) we derive an extinction-free surface flux of $8.810^{4} \mathrm{~W} \mathrm{~m}^{-2}$ for the emission-component of the $\mathrm{Mg} \mathrm{II} \mathrm{k}$ line of BN Ori. In the same way we derive from the corresponding profile of HR 5999 in the phase of maximum visual brightness (Blondel et al. 1989) an extinction-free surface flux of $6.4510^{6} \mathrm{~W} \mathrm{~m}^{-2}\left(V^{\mathrm{J}}=6 . \mathrm{m}^{\mathrm{m}} 8\right)$ and $1.210^{7} \mathrm{~W} \mathrm{~m}^{-2}\left(V^{\mathrm{J}}\right.$ $\left.=7{ }^{\mathrm{m}} 6\right)$. The ratio of the $\mathrm{Mg} \mathrm{II} \mathrm{k}$ emission fluxes of HR 5999 and BN Ori is therefore close to $70-140$. This is higher than the ratio of the $\mathrm{H} \alpha$ fluxes of the two stars (Sect. 4).

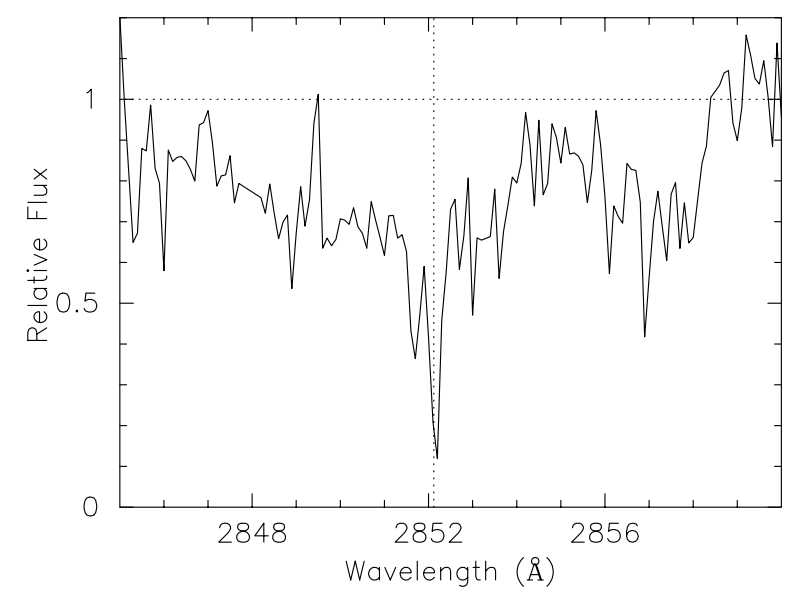

Fig. 11. Mg I $2852 \AA$ components of BN Ori

Table 9. Comparion of emission fluxes $\left(\mathrm{W} \mathrm{m}^{-2}\right)$

\begin{tabular}{lrrrrr}
\hline \multicolumn{1}{c}{ star } & \multicolumn{1}{c}{$\mathrm{H} \alpha$} & $\mathrm{Mg} \mathrm{II}$ & $\frac{\mathrm{Mg} \mathrm{II}}{\mathrm{H} \alpha}$ & $V^{\mathrm{J}}$ \\
\hline BN Ori & $1.6-1.210^{5}$ & $8.810^{4}$ & 0.63 & 9.7 \\
FU Ori & $3.810^{5}$ & $1.710^{6}$ & 4.4 & 9.3 \\
HR 5999 & $3.4-2.310^{6}$ & $6.510^{6}$ & 2.4 & 6.8 \\
HR 5999 & $5.810^{6}$ & $1.210^{7}$ & 2.1 & 7.6 \\
\hline
\end{tabular}

The high-resolution Mg II profiles of FU Ori have been published by Ewald et al. (1986). The profiles have a P-Cygni shape, but the noise in the neighbouring continuum does not permit to determine an outflow velocity. The extinction-corrected surface flux in the symmetrised $\mathrm{Mg}$ II k emission-profile is $8.610^{7} \mathrm{erg} \mathrm{cm}^{-2} \mathrm{~s}^{-1}$ and the corresponding flux of the $\mathrm{Mg}$ II h emission is $7.110^{7} \mathrm{erg} \mathrm{cm}^{-2} \mathrm{~s}^{-1}$. This total Mg II emission flux of FU Ori is close to the value obtained by Ewald et al. With the use of the observed Mg II profile of the F0 Ib star $\alpha$ Car (Praderie et al. 1980), which has a $v \sin i=$ $0 \mathrm{~km} \mathrm{~s}^{-1}$, we corrected the profile of FU Ori for the photospheric contribution. The extinction-corrected surface-flux of the symmetrised Mg II k-profile then becomes $1.6710^{6} \mathrm{~W} \mathrm{~m}^{-2}$. Table 9 gives a survey of the emission fluxes of the 3 stars. Although the $\mathrm{H} \alpha$ and Mg II profiles show variations on a short time-scale $(\sim 1$ d $)$ the time variations in the integrated emission fluxes are small. This gives us a justification to use Table 9 in the following discussion, in spite of the fact that the $\mathrm{H} \alpha$ and $\mathrm{Mg}$ II fluxes were not observed simultaneously.

\section{Discussion}

The light curve of BN Ori and its spectral type A7 (Cannon 1931) during the first half of the century suggest that in this period BN Ori was a Herbig Ae star with strong brightness-variations due to a variable circumstellar dust extinction. The recent detection of emission from a nearby reflection nebulosity (Sect. 3) and the value of the stellar mass (Sect. 5) supports its previous membership to this class of intermediate-mass pre-main-sequence stars, first introduced by Herbig (1960b).

Around 1947 the large-scale light curve of BN Ori changed drastically in a way which is similar to the transition in the light curves of classical FUORs. In particular the light curve of BN Ori has much in common with that of the FUOR V1515 Cyg due to its last active epoch of variations in brightness with the further rise typical for FUORs, a delay at the maximum phase and specifically a typical short minimum after the first maximum (Fig. 12). The sequence and scale of the events in the pre-maximum and post-maximum phases of the BN Ori light curve are also consistent with those of other classical FUORs. The only possible difference with the FUOR light curves is the large amplitude of the variability in the pre-maximum epoch of BN Ori. The light-variation level of the classical FUORs was less in this phase, but for the lack of any reliable information it cannot be argued that the range of brightness-variations in the pre-flare phase of FUORs was small. Therefore, as far as the light curve is concerned, we have every reason to consider BN Ori as an object which is related to FUORs.

The spectroscopic observations of BN Ori, described in Sects. 4 and 5, show that there are also spectral similarities between BN Ori and the classical FUORs, such as FU Ori:

1. The various spectral classifications of BN Ori by successive observers: A7 (Cannon 1931; Hoffmeister 1949; Payne-Gaposhkina 1952), A6 - A8 (Herbig 1954, 1960a), F0 (Zajtseva 1970) and F2 - F3 (Kolotilov \& Zajtseva 1976) could raise the impression that BN Ori has changed to progressively later spectral types in the course of the years. However, as already suspected by Herbig (1960a), several spectral types can be present simultaneously in the spectrum of BN Ori. Our spectra (Sect. 4) show a variety of spectral types ranging from 


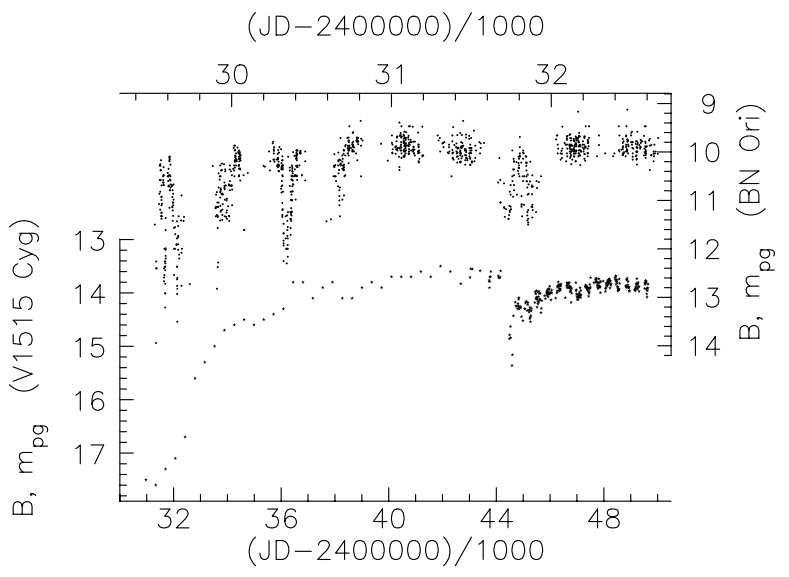

Fig. 12. Comparison of the light curves of BN Ori (upper part; dots) and the FUOR V1515 Cyg (lower part; stars)

A6 - A 8 from the higher Balmer terms, A8 - F0 from the Ca IIK line, F0 - F2 from the G-band, F0 - G5 from the Fe I lines, F6 - G0 from the O I (1) IR-triplet G5 from the CaII (2) IR-triplet until late-G to early$\mathrm{K}$ from the strength of the Mn I triplet around $4033 \AA$. This suggests that the atmosphere of BN Ori is thermally stratified in the sense that it consists of an A6 - A7 photosphere, surrounded by a cooler envelope. Such a thermal stratification is also a characteristic feature of FUOR atmospheres, e.g. FU Ori has a photosphere of type F2:pI - IIe (Herbig 1966), estimated from the Balmer lines and the low excitation lines of neutral and singly-ionised metals in the visual, an envelope (or disc) of type $\mathrm{K}$ from the Ca II (2) IR-triplet and a type $\mathrm{K}-\mathrm{M}$ from the $\mathrm{CO}$ bands in the NIR part of the spectrum. However, in contrast to the situation for BN Ori and for FU Ori we find only a small range of spectral types, A5 - A7 III (Bessel \& Eggen 1972; Tjin A Djie et al. 1989), for the visual and red parts of the spectrum of the Herbig Ae star HR 5999, which suggests a much lower degree of stratification of the atmosphere of this star.

2. Another similarity with classical FUORs is the presence of high-velocity outflow-components in the $\mathrm{H} \alpha$ P-Cygni type profiles: $-680 \mathrm{kms}^{-1}$ for BN Ori and $-550 \mathrm{~km} \mathrm{~s}^{-1}$ for FU Ori. The time-scale of the shape-variability of this profile may be less than $24^{\mathrm{h}}$ (Kolotilov \& Zajtseva 1976) for BN Ori, which is comparable with those observed for FUORs. This suggests that the line is formed in a region of small extension. The large width of the emission-component profile (Fig. 7) indicates that this region is within a distance of $1 R_{\star}$ from the stellar surface. Similar to the fast winds from FUORs (Croswell et al. 1987), the fast outflow from BN Ori is probably driven by strong magnetic fields, originating near the accretion-disc boundary layer (Pringle 1989). In contrast with this, we find only relatively low-outflow velocity-components in the $\mathrm{H} \alpha$-profiles of HR 5999: in general $\sim-50 \mathrm{~km} \mathrm{~s}^{-1}$ and occasionally $\sim-120 \mathrm{kms}^{-1}$ (Tjin A Djie et al. 1989). The time-scale of the $\mathrm{H} \alpha$ shape-variability for $\mathrm{HR} 5999$ is of the same order as that of BN Ori. The profiles of $\mathrm{Mg}$ II h\&k are similar in shape to those of $\mathrm{H} \alpha$ in the three stars. The velocity of the outflow-components in the $\mathrm{Mg}$ II profiles seems to be lower than that in $\mathrm{H} \alpha\left(\sim-250 \mathrm{~km} \mathrm{~s}^{-1}\right.$ for BN Ori) but these values are rather uncertain, due to the low $\mathrm{S} / \mathrm{N}$ for the neighbouring continuum. Since only one high-resolution UVspectrum has been obtained for BN Ori and two for FU Ori, the time-scales of variability of the Mg II profiles are unknown.

3. Several prominent photospheric lines in the spectra of FU Ori and the FUOR BBW 76 (Reipurth 1990): Li I $(6708 \AA)$ and Ba II (6497 $\AA$ ) are also prominently present in the spectrum of BN Ori (Sect. 4.3) with comparable strength. In the spectrum of HR 5999, however, the Li I line is missing.

Besides these similarities between the spectrum of BN Ori and those of the classical FUORs there are also differences:

1. The spectrum of BN Ori is of luminosity-class III-IV while the FUOR spectra have luminosity-classes I-II.

2. The rotation rate $v \sin i$ of BN Ori is about $220 \mathrm{~km} \mathrm{~s}^{-1}$, which is close to those of several intermediate-mass Herbig Ae stars ( $v \sin i=180 \mathrm{~km} \mathrm{~s}^{-1}$ for HR 5999). In contrast with this, the rotation rates of classical FUORs are in the range of $60 \mathrm{~km} \mathrm{~s}^{-1}$ (FU Ori) which is more similar to those of their $\mathrm{T}$ Tauri precursors.

3. BN Ori has a more extended shell than FU Ori. This is illustrated by the FW distribution over the excitation energies $\left(E_{\chi}\right)$ of Tables 6,11 and 8 . If we limit ourselves to the unblended lines of these tables and if we can consider the FWs to be proportional to the $v \sin i$ of the regions of formation of these lines we can, assuming a Kepler motion, estimate mean distances of these regions to the stellar surface. The values of $M_{\star}$ and $R_{\star}$ needed for this transformation were taken from Table 7 . The distribution of $E_{\chi}$ (of the lower-level) versus the mean Kepler distance of the line-forming regions (in $R_{\star}$ ) is given in Fig. 13 for BN Ori, FU Ori and HR 5999. For comparison the thermal stratification of the FUOR V1057 Cyg is indicated by the dotted line. Welty et al. (1990) showed that this stratification originates from the differential rotation in the disk. Figure 13 demonstrates that outside the stratified envelope there is an extended shell region for BN Ori, FU Ori and HR 5999. This is especially clear from the Fe II \& Cr II absorption lines (with lower level $E_{\chi} \leq 1 \mathrm{eV}$ ) which are still formed far away from the star $\left(\sim 40 R_{\star}\right.$ for FU Ori and HR 5999, and $>400 R_{\star}$ for BN Ori). The difference of the shell extension is illustrated by the least-square fits for BN Ori and HR 5999 in Fig. 13. The uncertainty for the size 


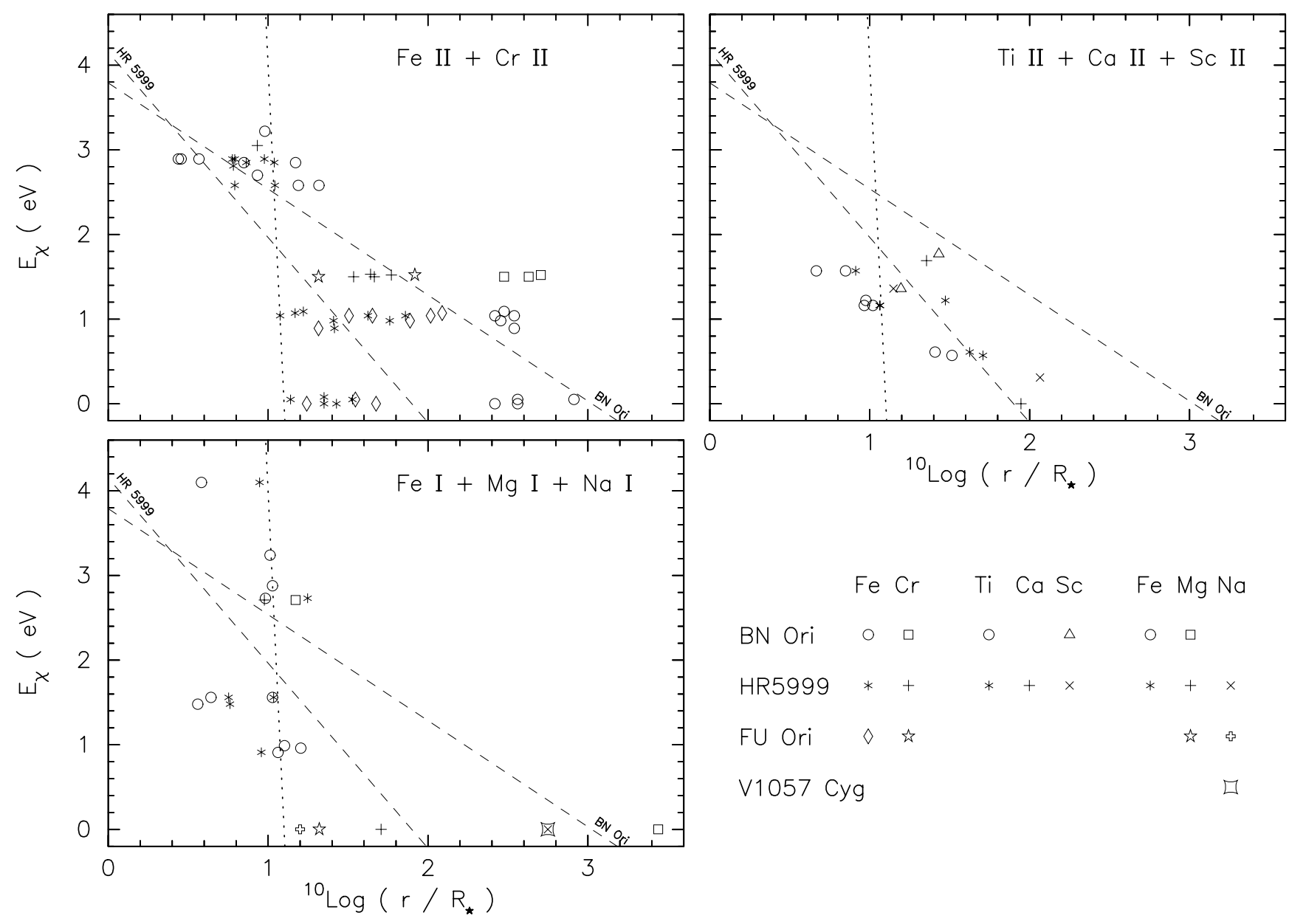

Fig. 13. Shell regions of BN Ori, HR 5999 and FU Ori; $E_{\chi}$ vs. distance to the surface (in units of $R_{\star}$ ). The two dashed lines are the least-square lines for BN Ori and HR 5999, using the values of the Fe II and Cr II lines together. The dotted line indicates the stratification of V1057 Cyg

of the BN Ori shell is due to the fact that the UVlines are so narrow that the widths may be dominated by Doppler broadening and therefore only give an upper limit to the distances. Although the cool shell of BN Ori extends much further out than those of FU Ori and HR 5999 we note from Table 8 that for each line: $\mathrm{EW}_{\mathrm{HR}}>\mathrm{EW}_{\mathrm{FU}}>\mathrm{EW}_{\mathrm{BN}}$. As the corresponding column densities follow the same order (BN:FU:HR = $1.0: 4.0: 8.6)$, this means that BN Ori has the most diluted shell of these 3 stars. This suggests that if BN Ori had a Herbig Ae star (like HR 5999) as its precursor, the thick shell has been expanded (to the shell remnant now present) as a result of a (FUOR) outburst. This expansion had a cooling influence on the extended Mg II emission region and less (or no) influence on the $\mathrm{H} \alpha$ emission region which is confined to a region closer to the star. The ratios in Table 9 are consistent with this scenario.
4. FU Ori and HR 5999 have very large excess fluxes in the NIR. These excesses are too large to be accounted for by the contribution of the accretion-discs only and therefore indicate the presence of significant quantities of radiating nearby circumstellar dust. Another indication for this are the large circumstellar colour excesses in Table 7. In contrast with this we find a very small NIRE and colour excess for BN Ori, which suggests an almost complete absence of circumstellar dust around this star.

After this comparison of the light curves and spectra of BN Ori, FU Ori and HR 5999 we propose the following scenario for the behaviour of BN Ori: The precursor of BN Ori was a Herbig A6 - A7e star, similar to HR 5999 but of somewhat lower mass (comparable with BF Ori) and with a slightly higher rotation rate. Around 1947 a FUOR type outburst occurred in the star-disc system with the result that the envelope around the inner part of the 
accretion-disc expanded. Except for the part near the star where most of the $\mathrm{H} \alpha$ and $\mathrm{Mg}$ II emission originates, the optically thin (shell) region at the outside of this envelope was expelled together with the outer part $\left(R>2.0 R_{\star}\right)$ of the accretion-disc. In the spectrum of FU Ori the shell components of the lines indicate that the shell moves outward with a velocity around $-50 \mathrm{~km} \mathrm{~s}^{-1}$ (Herbig 1966), but for BN Ori we have no radial-velocity measurements available. Most of the circumstellar dust was probably blown away after the break-up of the outer part of the magnetic field, which confined the dust to the circumstellar region (Il'in \& Voshchinnikov 1993) before the outburst. The disappearance of this dust (or the smoothing of its distribution), may explain the levelling-off of the light curve after the outburst.

This scenario raises several questions, the most important being whether a FUOR outburst can occur in a Herbig Ae star. Recently Bell et al. (1995) have shown that recurrent FUOR outburst can occur in young stars with disc accretion as a result of a thermal-runaway in the inner part of the accretion-disc when the mass-accretion rate rises above a certain critical value. For $2-3 M_{\odot}$ pre-main-sequence stars, such as the Herbig stars, Bell (1994) predicted that this critical value is around a few times $10^{-7} M_{\odot} \mathrm{yr}^{-1}$. It may be interesting to note that from matching the low-dispersion UV-spectra of BN Ori, BF Ori and HR 5999 we found mass-accretion rates of $0.18,3.2$ and $5.410^{-7} M_{\odot} \mathrm{yr}^{-1}$ respectively. The latter two values are close to the critical value of the mass-accretion rate, while the low rate for BN Ori (compared e.g. with that of FU Ori) may be due to the present lack of supply of inflow from the circumstellar shell region.

If we accept the FUOR character of BN Ori we are faced with the question why BN Ori is different from the classical FUORs. Two main reasons for differences may be proposed: First of all there is the difference in mass and rotation rate between Herbig Ae and $\mathrm{T}$ Tauri precursors which could influence the relative violence of the outbursts. The present low accretion rate of BN Ori (corresponding to an optically thin disc) compared to that of FU Ori may permit a more rapid expansion of its outer shell, which could explain the difference mentioned in point 3 discussed above. Secondly, successive outbursts in a recurrent FUOR have differences in their initial conditions and may therefore show differences in their post-flare appearence. Recurrent outbursts in BN Ori would facilitate a gradual exhaustion of the shell supply, as well as the accumulative production of sizable amounts of Li I at the surface of BN Ori, e.g. by spallation reactions induced by high fluxes of energetic protons in the FUOR flares.

Acknowledgements. The authors would like to thank Dr. A. Brown (JILA) for collaborating in the IUE observations and reductions of BN Ori. Furthermore, we are indebted to all the observers mentioned in Tables 1, 5 and 12 and to Dr. J.R.W. Heintze (RUU) for his efforts to develop and maintain the photometric facilities at DASA. This research has made use of the
Simbad data base operated at CDS, Strasbourg, France and of the IUE archives operated by ESA at Villafranca del Castillo, Spain.

\section{References}

Ambartsumian V.A., 1971, Afz 7, 557 (Astroph. 7, 331)

Artyukhina N.M., 1959, AZh 36, 832 (1960, Sov. Astr. 3, 808)

Baade D., Stahl O., 1989, A\&A 209, 255

Bastian U., Mundt R., 1985, A\&A 144, 57

Barylak M., 1989, IUE ESA Newslett. 33, 20

Bell K.R., 1994, in The Nature and Evolutionary Status of Herbig Ae/Be Stars, Thé P.S., Pérez M.R., van den Heuvel E.P.J. (eds.), ASP Conf. Ser. 62, 215

Bell K.R., Lin D.N.C., 1994, ApJ 427, 987

Bell K.R., Lin D.N.C., Hartmann L.W. Kenyon S.J., 1995, ApJ 444, 376

Bessel M.S., Eggen O.J., 1972, AJ 177, 209

Beyer M., 1937, Astron. Nachr. 263, 67

Blondel P.F.C., Tjin A Djie H.R.E., Thé P.S., 1989, A\&AS 80, 115

Blondel P.F.C., Tjin A Djie H.R.E., 1994, in The Nature and Evolutionary Status of Herbig Ae/Be Stars, Thé P.S., Pérez M.R., van den Heuvel E.P.J. (eds.) ASP Conf. Ser. 62, 211

Blondel P.F.C., Tjin A DjieH.R.E., 1997 (in preparation)

Bouchet P., Monetti A., Slezak E., Le Bertre T., Manfroid J., 1989, A\&AS 80, 379

Bouchet P., Manfroid J., Schmider F.X., 1991, A\&AS 91, 409

Brand J., Wouterloot J.G.A., 1988, A\&AS 75, 117

Brown A., Tjin A DjieH.R.E., Thé P.S., 1986, in New Insights in Astrophysics, UCL, London, ESA SP-263, 173

Bychkov V.D., Vitrichenko E.A., Shcherbakov A.G., 1978, Izvestiya Krymskoi Astr. Obs. 58, 81

Cannon A.J., 1919, Harvard Circ. 218

Cannon A.J., 1931, Harvard Ann. 100, 87

Clarke C.J., Lin D.N.C., Papaloizou J.C.B., 1989, MNRAS 236,495

Cohen M., 1973, MNRAS 161, 97

Croswell K., Hartmann L., Avrett E.H., 1987, ApJ 312, 227

Doazan V., Sedmak G., Barylak M., Rusconi L., 1991, in A Be Star Atlas of far-UV and Optical High-resolution Spectra, Battrick B. (ed.) ESTEC Noordwijk, The Netherlands, ESA SP-1147

Dragomiretskaya B.A., 1965, Afz 1, 455 (Astroph. 1, 241)

Esch M., 1925, Valkenburg Ver. 2, 83

Ewald R., Imhoff C.L., Giampapa M.S., 1986, in New Insights in Astrophysics, UCL, London, ESA SP-263, 205

Faraggiana R., Gerbaldi M., van 't Veer C., Floquet M., 1988, A\&A 201, 259

Felenbok P., Praderie F., Talavera A., 1983, A\&A 128, 74

Felenbok P., Czarny J., Catala C., Praderie F., 1988, A\&A 201, 247

Fernández M., Ortiz E., Eiroa C., Miranda L.F., 1995, A\&AS 114,439

Garrison L.M., 1978, ApJ 224, 535

Glass I.S., Penston M.V., 1974, MNRAS 167, 237

Götz W., 1980, Mitt. Veränd. Sterne, B8, H9 143

Grady C.A., Pérez M.R., Talavera A., et al., 1996, A\&AS (submitted)

Hamann F., Persson S.E., 1992, ApJS 82, 285 
Hartmann L., Kenyon S.J., Hewett R., et al., 1989, ApJ 338, 1001

Herbig G.H., 1954, Trans. IAU 8, 806

Herbig G.H., 1960a, ApJ 131, 632

Herbig G.H., 1960b, ApJS 4, 337

Herbig G.H., 1966, Vistas Astron. 8, 109

Herbig G.H., 1977, ApJ 217, 693

Hobbs L.M., 1974, ApJ 191, 381

Hoffmeister C., 1949, Astron. Nachr. 278, 24

Il'in V.B., Voshchinnikov N.V., 1993, AZh 70, 721 (Astr. Report 37, 362)

Ishchenko I.M., 1982, Per. Zv. 21, 665 (Var. Stars Moscow)

Kilyachkov N.N., Shevschenko V.S., 1976, Pis'ma AZh 2, 494 (1977, Sov. Astr. Let. 2, 193)

Kolotilov E.A., Zajtseva G.V., 1976, Afz 12, 31 (Astroph. 12, 23)

Kolotilov, E.A., Zajtseva, G.V., Shenavrin, V.I. 1977, Afz 13, 449 (Astroph. 13, 253)

Kopylov I.M., 1958, Izvestiya Krymskoi Astr. Obs. 20, 123

Kovalchuk G.U., 1991, IAU Inf. Bull. Var. Stars 3582, 1

Kurucz R.L., 1979, ApJS 40, 1

Kurucz R.L., 1991, in Precision photometry: Astrophysics of the galaxy, Davis Philip A.G., Upgren A.R., Janes K.A. (eds.). L. Davis press, Schenectady, New York

Lub J., Pel J.W., 1977, A\&A 54, 137

Martinov D.Ya., 1951, Publ. Engelhardt Obs. (Kazan Univ.) 26,17

Palla F., Stahler S.W., 1992, ApJ 392, 667

Palla F., Stahler S.W., 1993, ApJ 418, 414

Parenago P.P., 1933, Veränd. Sterne IV No. 7 (43), 221

Payne-Gaposhkina C., 1952, Ann. Harv. Coll. Obs. 118, No. 3

Praderie F., Talavera A., Lamers H.J.G.L.M., 1980, A\&A 86, 271

Praderie F., Catala C., Czarny J., Thé P.S., Tjin A Djie H.R.E., 1991, A\&AS 89, 91

Pringle J.E., 1989, MNRAS 236, 107

Reipurth B., 1990, IAU Symp. 137, ESO Sc. Reprint 695

Savage B.D., Mathis J.S., 1979, ARA\&A 17, 73
Schmidt-Kaler Th., 1982, in: Landolt-Börnstein Tables Vol. VI/2b. Springer-Verlag

Shanin G.I., 1979, AZh 56, 288 (1979, Sov. Astr. 23(2), 158)

Shevchenko V.S., 1989, The Herbig Ae/Be stars, F.A.N. Publishers, Tashkent, Uzbekistan (in Russian)

Slettebak A., Collins IIG.W., Boyce P.B., White N.M., Parkinson T.D., 1975, ApJS 29, 137

Slettebak A., 1986, PASP 98, 867

Sterken C., Manfroid J., Anton K., et al., 1993, A\&AS 102, 79

Sterken C., Manfroid J., Beele D., et al., 1995, A\&AS 113, 31

Strömgren B., 1948, ApJ 108, 242

Tjin A Djie H.R.E., Thé P.S., Hack M., Selvelli P.L., 1982, A\&A 106, 98

Tjin A Djie H.R.E., Blondel P.F.C., 1997, A\&A (in preparation)

Tjin A Djie H.R.E., Thé P.S., Andersen J., Nordstrom B., Finkenzeller U., Jankovics I., 1989, A\&AS 78, 1

Tsesevich V.P., Dragomireskaya B.A., 1973, RW Aurigae Stars, Kiev (in Russian)

van den Ancker M.E., Thé P.S., de Winter D., 1996, A\&A 309, 809

Walker M.F., 1969, ApJ 155, 447

Wehrse R., 1974, A List of all Fraunhofer lines of the Rowland tables arranged by elements, Heidelberg

Wenzel W., 1968, IAU Coll. Non Periodic Phenomena in Variable Stars, Detre L. (ed.) Budapest, p. 61

Wenzel W., Dorschner J., Friedemann Chr., 1971, Astron. Nachr. 292 H5-6, 221

de Winter D., 1995 (private communication)

Zaidel A.N., Prokof'ev V.K., Raiskii S.M., Slavnyi V.A., Shreider E.Ya, 1969, Tables of Spectral lines, Nauka Press Moscow. Transl. IFI Plenum Press 1970 NewYork London Zajtseva G.V., 1968, Per. Zv. 16, 435 (Var. Stars Moscow)

Zajtseva G.V., 1970, Per. Zv. 17, 294 (Var. Stars Moscow)

Zajtseva G.V., 1971, Afz 7, 333 (Astroph. 7, 197)

Zajtseva G.V., Kolotilov E.A., 1973, Afz 9, 185 (Astroph. 9, 104) 
Table 10. Description of the symbols and references used in Tables 6,11 and 8

\begin{tabular}{|c|c|c|c|}
\hline $\begin{array}{l}\text { Nega } \\
\text { Italic }\end{array}$ & $\begin{array}{l}\text { ive EW-values in } \\
\text { EW-values indic }\end{array}$ & (the & $\begin{array}{l}\text { hission } \\
\text { ange of) variability }\end{array}$ \\
\hline Line & ources: & $\mathrm{i}$ & interstellar \\
\hline $\mathrm{p}$ & photospheric & $\mathrm{s}$ & shell, c.s. envelope \\
\hline $\mathrm{c}$ & chromospheric & $\mathrm{d}$ & disc \\
\hline Line & trengths: & $:$ & uncertain value \\
\hline $\mathrm{b}$ & blended & $\mathrm{m}$ & medium \\
\hline vs & very strong & $\mathrm{w}$ & weak \\
\hline $\mathrm{s}$ & strong & $\mathrm{vw}$ & very weak \\
\hline$\overline{\text { Refer }}$ & nces: & & \\
\hline 1 & BTA-spectrum & v. 21 & 1991 \\
\hline 2 & Kolotilov \& Za & eva ( & 76) \\
\hline 3 & Herbig (1966) & & \\
\hline 4 & Tjin A Djie et & $(198$ & \\
\hline 5 & Hamann \& Per: & n $(19$ & \\
\hline 6 & Felenbok et al. & $88)$ & \\
\hline 7 & $\mathrm{ESO}(1.5 \mathrm{~m})$ lor & esolu & on spectra Dec'92 \\
\hline 8 & ESO CAT high & solut & n spectra Dec'94, Jan'95 \\
\hline 9 & Reipurth (1990 & & \\
\hline 10 & Shanin (1979) & & \\
\hline
\end{tabular}

Table 11. Identification and source $(\boldsymbol{S})$ of lines in the high-resolution visual spectra of BN Ori, HR 5999 and FU Ori. The equivalent widths $(\mathrm{EW})$ in $(\AA)$ and full widths at half maximum $(\mathrm{FW})$ in $\left(\mathrm{km} \mathrm{s}^{-1}\right)$ are also given. Identification of the lines has been made with the help of the Tables of Zaidel et al. (1969) and excitation energies $\left(E_{\chi}\right)$ have been taken from Wehrse (1974). See Table 10 for the description of the used symbols

\begin{tabular}{|c|c|c|c|c|c|c|c|c|}
\hline \multirow{2}{*}{$\begin{array}{c}\lambda_{0} \\
(\AA)\end{array}$} & \multirow[b]{2}{*}{ Line id. } & $E_{\chi}$ & & \multicolumn{2}{|c|}{ BN Ori } & \multicolumn{2}{|c|}{ HR 5999} & \multirow{2}{*}{$\begin{array}{c}\text { FU Ori } \\
\mathrm{EW}^{3}\end{array}$} \\
\hline & & $(\mathrm{eV})$ & $S$ & $\mathrm{EW}^{1}$ & FW & $\mathrm{EW}^{4}$ & FW & \\
\hline 3608.86 & Fe I $(23)$ & 1.01 & & 0.09 & 193 & - & - & - \\
\hline 3721.94 & Н 14 & 10.20 & $\mathrm{p}$ & 1.62 & - & 1.70 & - & - \\
\hline 3727.03 & Fe I $(668)$ & 3.27 & & - & - & - & - & - \\
\hline 3727.20 & Cr II (117) & 4.78 & & - & - & - & - & - \\
\hline 3734.37 & H 13 & 10.20 & $\mathrm{p}$ & 2.37 & - & 1.17 & - & - \\
\hline 3741.63 & Ti II (72) & 1.58 & & - & - & 0.04 & - & - \\
\hline 750.15 & H 12 & 10.20 & $\mathrm{p}$ & 3.54 & - & 2.44 & - & - \\
\hline 3759.29 & Ti II (13) & 0.61 & $\mathrm{~s}$ & $0.37 \mathrm{~b}$ & 130 & 0.74 & 110 & - \\
\hline 3761.32 & Ti II (13) & 0.57 & $\mathrm{~s}$ & $"$ & - & 0.64 & 100 & - \\
\hline 3770.63 & H 11 & 10.20 & $\mathrm{p}$ & 3.01 & - & 3.5 & - & - \\
\hline 3797. & H 10 & 10.20 & $\mathrm{p}$ & 3.83 & - & 6.0 & - & - \\
\hline 3813.39 & Ti II (12) & 0.61 & $\mathrm{~s}$ & $0.20 \mathrm{~b}$ & 310 & $0.48 \mathrm{~b}$ & 160 & - \\
\hline 3814.12 & Fe II (153) & 4.74 & & $"$ & - & $"$ & - & - \\
\hline 3814.58 & Ti II (12) & 0.57 & $\mathrm{~s}$ & $"$ & - & $"$ & - & - \\
\hline 3815.25 & Fe I $(45)$ & 1.48 & & $"$ & - & $"$ & - & - \\
\hline 3835.38 & Н 9 & 10.20 & $\mathrm{p}$ & 7.41 & - & 9.1 & - & - \\
\hline 3872.51 & Fe I $(20)$ & 0.99 & & 0.38 & 209 & 0.27 & - & - \\
\hline 3889.05 & H 8 & 10.20 & ps & 6.19 & - & 10.6 & - & - \\
\hline 3905.53 & Si I （3) & 1.91 & & - & - & - & - & - \\
\hline 3906.04 & Fe II (173) & 5.57 & & - & - & - & - & - \\
\hline
\end{tabular}

Table 11. continued

\begin{tabular}{|c|c|c|c|c|c|c|c|c|}
\hline \multirow{2}{*}{$\begin{array}{c}\lambda_{0} \\
(\AA)\end{array}$} & \multirow[b]{2}{*}{ Line id. } & \multirow{2}{*}{$\begin{array}{c}E_{\chi} \\
(\mathrm{eV})\end{array}$} & \multirow[b]{2}{*}{$S$} & \multicolumn{2}{|c|}{ BN Ori } & \multicolumn{2}{|c|}{ HR 5999} & \multirow{2}{*}{$\begin{array}{c}\text { FU Ori } \\
\mathrm{EW}^{3}\end{array}$} \\
\hline & & & & $\mathrm{EW}^{1}$ & FW & $\mathrm{EW}^{4}$ & FW & \\
\hline 3906.48 & Fe I $(4)$ & 0.11 & & - & - & - & - & - \\
\hline 3933.67 & Ca II K (1) & 0.00 & $\mathrm{pc}$ & 5.45 & 440 & 4.1 & 76 & st \\
\hline 3944.01 & $\mathrm{Al}$ I ( 1$)$ & 0.00 & ps & 0.09 & 160 & 0.1 & 152 & st \\
\hline 3968.47 & CaII & 0.00 & $\mathrm{pc}$ & 7.70 & 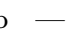 & $12.5 \mathrm{l}$ & $b-$ & st \\
\hline 3970.07 & $\mathrm{H} \varepsilon$ & 10.20 & ps & $"$ & - & $"$ & - & st \\
\hline 3983.96 & Fe I $(277)$ & 2.73 & & - & - & - & - & - \\
\hline 3997.39 & Fe I (278) & 2.73 & & 0.17 & 240 & 0.17 & 170 & - \\
\hline 4005.23 & Fe I (43) & 1.56 & $\mathrm{p}$ & 0.29 & 355 & 0.28 & 300 & - \\
\hline 4014.53 & Fe I (802) & 2.57 & & - & - & - & - & - \\
\hline 4025.13 & Ti II (11) & 0.61 & & - & - & - & - & - \\
\hline 4030.76 & $\operatorname{MnI}(2)$ & 0.00 & & 0.78 & 391 & 0.601 & - & - \\
\hline 4033.07 & $\operatorname{MnI}(2)$ & 0.00 & & $"$ & - & $"$ & - & - \\
\hline 4034.49 & Mn I ( 2) & 0.00 & & $"$ & - & $"$ & - & - \\
\hline 4035.67 & V II $(216)$ & 1.79 & & $"$ & 一 & $"$ & - & - \\
\hline 4045.82 & Fe I (43) & 1.48 & ps & 0.41 & 390 & 0.50 & 297 & st \\
\hline 4053.81 & Ti II (87) & 1.89 & & - & - & - & - & $\mathrm{vw}$ \\
\hline 4057.36 & Fe I (277) & 2.76 & & - & - & - & - & - \\
\hline 4057.50 & Mg I (10) & 4.34 & & - & - & - & - & - \\
\hline 4063.28 & Fe I (698) & 3.37 & & 0.20 & о 389 & $0.27 \mathrm{I}$ & b 295 & $\mathrm{~m}$ \\
\hline 4063.60 & Fe I (43) & 1.61 & $\mathrm{p}$ & $"$ & - & $"$ & - & $\mathrm{vw}$ \\
\hline 4077.72 & Sr II ( 1$)$ & 0.0 & ps & 0.30 & 290 & 0.34 & 260 & st \\
\hline 4085.31 & Fe I (559) & 3.24 & & 0.07 & 232 & - & - & - \\
\hline 4101.74 & & 10.20 & ps & 3.77 & - & 11.2 & - & st \\
\hline 4118.55 & Fe I (801) & 3.67 & & 0.09 & - & - & - & - \\
\hline 4134.20 & Fe I (217) & 2.61 & & 0.10 & - & - & - & $\mathrm{w}$ \\
\hline 4143.76 & Fe I (43) & 1.56 & & 0.18 & 228 & 0.18 & 217 & - \\
\hline 4154.50 & Fe I (355) & 2.03 & & 0.24 & 110 & 0.211 & b 289 & - \\
\hline 4154.81 & Fe I (694) & 3.37 & & $"$ & - & $"$ & - & - \\
\hline 4156.78 & Fe I (354) & 2.88 & & 0.24 & 110 & - & - & - \\
\hline 4173.45 & FeII $(27)$ & 2.58 & $\mathrm{~s}$ & 0.26 & 189 & 0.38 & 287 & - \\
\hline 4177.55 & Y II (14) & 0.41 & $\mathrm{~s}$ & 0.18 & 200 & 0.361 & b 251 & - \\
\hline 4177.60 & Fe I (18) & 0.91 & & $"$ & - & $"$ & - & - \\
\hline 4178.87 & Fe II $(28)$ & 2.58 & $\mathrm{~s}$ & $"$ & - & $"$ & - & - \\
\hline 4187.04 & Fe I (152) & 2.45 & & - & - & $0.17 \mathrm{l}$ & b 287 & - \\
\hline 4187.80 & Fe I (152) & 2.42 & & - & - & $"$ & - & - \\
\hline 4202.76 & Fe I $(42)$ & 1.48 & & - & - & 0.14 & - & - \\
\hline 4215.52 & Sr II ( 1$)$ & 0.00 & ps & 0.07 & 250 & 0.29 & 270 & st \\
\hline 4226.73 & $\mathrm{Ca}$ I (2) & 0.00 & ps & 0.53 & 240 & 0.60 & 260 & st \\
\hline 4233.17 & FeII $(27)$ & 2.58 & $\mathrm{~s}$ & 0.16 & 163 & 0.36 & 215 & - \\
\hline 4246.83 & Sc II $(7)$ & 0.31 & ps & - & - & 0.20 & 265 & $\mathrm{~m}$ \\
\hline 4252.62 & Cr II (31) & 3.86 & & - & - & 0.061 & b 88 & - \\
\hline 4254.33 & Cr I (1) & 0.00 & & - & - & $"$ & - & st \\
\hline 4261.90 & Cr II (31) & 3.86 & & - & 190: & $0.25 \mathrm{l}$ & b 230 & - \\
\hline 4262.13 & Cr I $(178)$ & 3.11 & & - & - & $"$ & - & - \\
\hline 4271.76 & Fe I $(42)$ & 1.49 & & 0.60 & o 444: & $1.28 \mathrm{l}$ & b 350: & - \\
\hline 4273.32 & FeII $(27)$ & 2.70 & & $"$ & - & $"$ & - & - \\
\hline 4274.81 & Cr I (1) & 0.00 & ps & $"$ & - & - & - & $\mathrm{m}$ \\
\hline 4284.21 & Cr II (31) & 3.05 & & - & - & 0.10 & 244 & - \\
\hline 4290.22 & Ti II (41) & 1.16 & & 0.18 & 245 & 0.32 & 210 & - \\
\hline 4301.93 & Ti II (41) & 1.16 & & 0.51 & o $370:$ & 0.561 & b 366: & - \\
\hline 4307.91 & Ti II (41) & 1.16 & $\mathrm{p}$ & 0.31 & 230 & 0.21 & 210 & $\mathrm{w}$ \\
\hline 4314.08 & Sc II $(15)$ & 0.62 & & 0.23 & $256:$ & $0.24 \mathrm{l}$ & b 220 & - \\
\hline 4314.29 & FeII $(32)$ & 2.68 & & $"$ & - & $"$ & - & - \\
\hline 4314.98 & Ti II (41) & 1.16 & & $"$ & - & $"$ & - & - \\
\hline
\end{tabular}


Table 11. continued

Table 11. continued

\begin{tabular}{|c|c|c|c|c|c|c|c|c|c|c|c|c|c|c|c|c|c|}
\hline \multirow{2}{*}{$\begin{array}{l}\lambda_{0} \\
(\AA)\end{array}$} & \multirow{2}{*}{\multicolumn{2}{|c|}{ Line id. }} & \multirow{2}{*}{$\begin{array}{c}E_{\chi} \\
(\mathrm{eV})\end{array}$} & \multirow[b]{2}{*}{$S$} & \multicolumn{2}{|c|}{ BN Ori } & \multicolumn{2}{|c|}{ HR 5999} & \multirow{2}{*}{$\begin{array}{cc}\text { FU Ori } & \lambda_{0} \\
\mathrm{EW}^{3} & (\AA)\end{array}$} & \multirow[b]{2}{*}{ Line id. } & \multirow{2}{*}{$\begin{array}{c}E_{\chi} \\
(\mathrm{eV})\end{array}$} & \multirow[b]{2}{*}{$S$} & \multicolumn{2}{|c|}{ BN Ori } & \multicolumn{2}{|c|}{ HR 5999} & \\
\hline & & & & & $\mathrm{EW}^{1}$ & FW & $\mathrm{EW}^{4}$ & FW & & & & & $\mathrm{EW}^{1}$ & FW & $\mathrm{EW}^{4}$ & FW & $\mathrm{EW}^{3}$ \\
\hline 4325.01 & Sc II & II $(15)$ & 0.60 & & $0.32 \mathrm{~b}$ & 330 & - & - & -5031.02 & Sc II $(23)$ & 1.36 & & 0.11 & 188 & - & 190 & - \\
\hline 4325.76 & $\mathrm{Fe} I$ & $I \quad(2)$ & 0.00 & & $"$ & - & - & - & -5041.08 & Fe I (16) & 0.96 & & $0.14 \mathrm{~b}$ & 240 & $0.33 \mathrm{~b}$ & 309 & - \\
\hline 4340.47 & & & 10.20 & ps & 3.95 & - & 11.8 & - & vs 5041.63 & $\mathrm{Ca}$ I (34) & 2.71 & & $"$ & - & $"$ & - & - \\
\hline 4354.76 & Fe II & II $(27)$ & 2.70 & & $0.18:$ & 254 & - & - & -5041.76 & $\mathrm{Fe}$ I $(36)$ & 1.48 & & $"$ & - & $"$ & - & - \\
\hline 4374.45 & Sc II & II $(14)$ & 0.62 & & $0.16 \mathrm{~b}$ & 250 & $0.19 \mathrm{~b}$ & 190 & -5051.64 & Fe I (16) & 0.91 & & 0.09 & 219 & 0.19 & 237 & - \\
\hline 4374.82 & Ti II & II $\quad(93)$ & 2.06 & & $"$ & - & - & - & -5079.23 & Fe I (66) & 2.20 & & $0.10 \mathrm{~b}$ & - & $0.08 \mathrm{~b}$ & - & - \\
\hline 4374.94 & YII & I (13) & 0.41 & & $"$ & - & $"$ & - & -5079.74 & Fe I (16) & 0.99 & & $"$ & - & $"$ & - & - \\
\hline 4383.55 & Fe I & (41) & 1.48 & & $0.65 \mathrm{~b}$ & 360 & $0.90 \mathrm{~b}$ & 274 & -5083.34 & Fe I $(16)$ & 0.96 & & 0.20 & 186 & - & - & $\mathrm{vw}$ \\
\hline 4384.21 & Fe II & II $\quad(32)$ & 2.66 & & $"$ & - & $"$ & - & -5098.70 & Fe I (66) & 2.18 & & $0.12 \mathrm{~b}$ & 124 & $0.13 \mathrm{~b}$ & - & - \\
\hline 4385.38 & Fe II & II $(27)$ & 2.78 & & $"$ & - & $"$ & - & -5100.66 & FeII (35) & 2.81 & & $"$ & - & $"$ & - & - \\
\hline 4394.06 & Tili & II $(51)$ & 1.22 & & $0.14 \mathrm{~b}$ & 233 & $0.30 \mathrm{~b}$ & 170 & -5139.26 & FeI $(383)$ & 3.00 & & $0.11 \mathrm{~b}$ & 184 & $0.13 \mathrm{~b}$ & - & - \\
\hline 4395.03 & Ti II & II $\quad(19)$ & 1.08 & ps & $"$ & - & $"$ & - & st 5139.47 & Fe I $(383)$ & 2.94 & & $"$ & - & $"$ & - & - \\
\hline 4395.83 & Ti II & I $(61)$ & 1.24 & & $"$ & - & $"$ & - & -5167.33 & $\operatorname{Mg}$ I ( 2) & 2.71 & & $-b$ & - & $-b$ & - & - \\
\hline 4416.82 & Fe II & I $(27)$ & 2.70 & & $0.33 \mathrm{~b}$ & 285 & - & 238 & -5169.03 & Fe II $(42)$ & 2.89 & ps & $0.80: \mathrm{b}$ & 387 & $0.72:$ & $232+116$ & - \\
\hline 4417.72 & Ti II & I $(40)$ & 1.16 & & $"$ & - & - & - & -5172.68 & $\operatorname{Mg}{ }_{1}(2)$ & 2.71 & & $0.47:$ & 193 & 0.36 : & 232 & $\mathrm{vw}$ \\
\hline 4434.96 & $\mathrm{Ca} \mathrm{I}$ & I $(4)$ & 1.89 & & $0.24 \mathrm{~b}$ & 286 & $0.15 \mathrm{~b}$ & 220 & -5208.44 & Cr I (7) & 0.04 & & $0.20 \mathrm{~b}$ & 121 & - & - & - \\
\hline 4435.10 & Fe I & $(2)$ & 0.09 & & $"$ & - & $"$ & - & -5208.60 & Fe I $(553)$ & 3.24 & & $"$ & - & - & - & - \\
\hline 4435.69 & $\mathrm{Ca} \mathrm{I}$ & I $(4)$ & 1.89 & & $"$ & - & $"$ & - & -5226.56 & Ti II (70) & 1.57 & & $0.33 \mathrm{~b}$ & 271 & - & - & $\mathrm{vw}$ \\
\hline 4443.80 & Tili & I $(19)$ & 1.08 & & $0.21 \mathrm{~b}$ & 214 & $0.25 \mathrm{~b}$ & - & -5226.87 & FeI (716) & 3.41 & & $"$ & - & - & - & - \\
\hline 4444.56 & Ti II & I $(31)$ & 1.12 & & $"$ & - & $"$ & - & -5234.62 & FeII (49) & 3.22 & & 0.31 & 241 & - & - & - \\
\hline 4459.12 & Fe I & $(68)$ & 2.18 & & - & - & - & - & -5264.80 & FeII (48) & 3.33 & & $1.38 \mathrm{~b}$ & - & - & - & - \\
\hline 4468.49 & Ti II & I $(31)$ & 1.13 & & - & - & $0.30 \mathrm{~b}$ & 201 & -5270.30 & Fe I (37) & 1.61 & & $"$ & - & - & - & - \\
\hline 4469.10 & Ti II & I $(18)$ & 1.08 & & - & - & $"$ & - & -5274.99 & Cr II (43) & 4.07 & & $0.58 \mathrm{~b}$ & - & - & - & - \\
\hline 4481.16 & Mg I & II $(4)$ & 8.89 & $\mathrm{p}$ & $0.34 \mathrm{~b}$ & 317 & $0.50 \mathrm{~b}$ & 250 & -5275.02 & Fe I (1029) & 4.07 & & $"$ & - & - & - & - \\
\hline 4481.33 & $\mathrm{Mg}$ I & II ( & 8.86 & $\mathrm{p}$ & $"$ & - & $"$ & - & -5316.61 & FeII $(48)$ & 3.22 & & $0.10 \mathrm{~b}$ & - & 0.32 & - & - \\
\hline 4491.40 & Fe II & I $(37)$ & 2.85 & & 0.25 & 280 & 0.22 & 264 & -5328.05 & Fe I (15) & 0.91 & & $0.70 \mathrm{~b}$ & - & $0.28 \mathrm{~b}$ & 168 & - \\
\hline 4508.28 & Fe II & I $(38)$ & 2.85 & & 0.04 & 193 & 0.18 & 216 & -5329.99 & FeI $(1028)$ & 4.07 & & $"$ & - & $"$ & - & - \\
\hline 4529.46 & Ti II & I $(82)$ & 1.57 & & 0.25 & 300 & 0.17 & 250 & -5341.02 & Fe I (37) & 1.61 & & 0.11 & 206 & 0.08 & - & - \\
\hline 4533.97 & Ti II & I $(50)$ & 1.24 & & $0.30 \mathrm{~b}$ & 300 & $0.32 \mathrm{~b}$ & 280 & -5364.88 & Fe I $(1146)$ & 4.44 & & 0.27 & 176 & 0.15 & 112 & - \\
\hline 4534.17 & Fe II & I $(37)$ & 2.85 & & $"$ & - & $"$ & - & -5371.50 & $\mathrm{Fe}_{\mathrm{I}}(15)$ & 0.96 & & $0.45 \mathrm{~b}$ & 293 & - & - & - \\
\hline 4549.47 & Fe II & I $(38)$ & 2.83 & ps & $0.19 \mathrm{~b}$ & 312 & $0.66 \mathrm{~b}$ & 363 & -5383.37 & Fe I (1140) & 4.31 & & 0.26 & 234 & - & - & - \\
\hline 4549.62 & Fe II & I $(82)$ & 1.58 & & $"$ & - & $"$ & - & -5397.13 & Fe I (15) & 0.91 & & $0.37 \mathrm{~b}$ & 116 & - & - & - \\
\hline 4555.89 & Fe II & I $\quad(37)$ & 2.83 & & $0.19 \mathrm{~b}$ & 277 & $0.40 \mathrm{~b}$ & - & -5405.78 & Fe I (15) & 0.99 & & $"$ & - & - & - & - \\
\hline 4558.66 & Cr II & II $(44)$ & 4.07 & & $"$ & - & $"$ & - & -5466.41 & Y II (12) & 1.43 & & 0.19 & 144 & - & - & - \\
\hline 4563.77 & Ti II & I $(50)$ & 1.22 & & 0.14 & 242 & 0.19 & 131 & -5476.91 & Ni I (597) & 1.83 & & 0.31 & 202 & - & - & - \\
\hline 4571.97 & Tili & I $(82)$ & 1.57 & & 0.18 & 330 & 0.12 & - & -5526.81 & Sc II $(31)$ & 1.77 & & 0.19 & 143 & - & - & - \\
\hline 4583.83 & Fe II & I $(38)$ & 2.81 & & - & - & 0.32 & 290 & -5535.48 & $\mathrm{Ba}$ I (2) & 0.00 & & 0.10 & 142 & 0.20 & - & - \\
\hline 4618.83 & Cr II & II $(44)$ & 4.07 & & - & - & 0.28 & 130 & -5586.76 & FeI $(680)$ & 3.37 & & 0.34 & 226 & - & - & - \\
\hline 4629.34 & Fe II & I $(37)$ & 2.81 & & - & - & 0.09 & 146 & -5638.27 & Fe I $(1087)$ & 4.22 & & $0.37 \mathrm{~b}$ & 171 & - & - & - \\
\hline 4670.17 & Fe II & I $(37)$ & 2.83 & & - & - & $0.31 \mathrm{~b}$ & 321 & -5644.14 & Ti I (240) & 2.27 & & $"$ & - & - & - & - \\
\hline 4670.40 & Sc II & I $(24)$ & 1.36 & & - & - & $"$ & - & -5658.83 & Fe I (686) & 3.40 & & $0.15 \mathrm{~b}$ & 167 & - & - & - \\
\hline 4861.33 & $\mathrm{H} \beta$ & & 10.20 & pcs & $3.0^{2}$ & - & 11.9 & - & -5662.16 & Ti I (249) & 2.32 & & 0.17 & 123 & - & - & - \\
\hline 4910.05 & $\mathrm{Fe} I$ & $(687)$ & 3.40 & & - & - & - & - & -5774.54 & Ti I (228) & 2.25 & & 0.19 & 137 & - & - & - \\
\hline 4911.21 & Ti II & I (114) & 3.12 & & - & - & - & - & -5785.98 & Ti I (309) & 3.32 & & 0.14 & 164 & $\mathrm{vw}$ & 100 & - \\
\hline 4923.92 & Fe II & I $(42)$ & 2.89 & ps & 0.53 & 449: & 0.64 & $292+107$ & -6053.48 & Cr II (105) & 4.74 & & 0.17 & 104 & - & - & - \\
\hline 4934.10 & Ba Il & II $(1)$ & 0.00 & & $0.78 \mathrm{~b}$ & - & - & - & -6136.62 & Fe I (169) & 2.45 & & $0.17 \mathrm{~b}$ & 154 & $0.22 \mathrm{~b}$ & - & - \\
\hline 4936.34 & Cr I & $(166)$ & 3.11 & & $"$ & - & - & - & -6137.70 & FeI (207) & 2.59 & & $"$ & - & $"$ & - & - \\
\hline 4957.30 & Fe I & (318) & 2.85 & & $0.28 \mathrm{~b}$ & - & $0.17 \mathrm{~b}$ & 193 & -6215.21 & Ti I (293) & 2.69 & & 0.22 & 152 & - & - & - \\
\hline 4957.61 & & $(318)$ & 2.81 & & 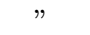 & - & $"$ & - & -6220.46 & Ti I (293) & 2.68 & & 0.11 & - & - & - & - \\
\hline 4982.51 & & (1867) & 4.10 & & $0.51 \mathrm{~b}$ & 380: & 0.23 & 240 & -6277.52 & Ti I (144) & 1.73 & & 0.48 & 200 & 0.33 & - & - \\
\hline 4984.13 & $\mathrm{Ni}$ I & $(143)$ & 3.80 & & $"$ & - & - & - & -6491.71 & Mn I (39) & 3.76 & & - & - & - & - & - \\
\hline 5005.72 & Fe I & $(984)$ & 3.08 & & $0.33 \mathrm{~b}$ & - & $0.31 \mathrm{~b}$ & 240 & -6494.98 & FeI (168) & 2.40 & & - & — & - & - & - \\
\hline 5006.12 & Fe I & (318) & 2.03 & & $"$ & - & $"$ & - & -6496.90 & BaII ( 2) & 0.60 & & 0.42 & 402 & 0.44 & 419 & vs \\
\hline 5018.43 & Fe II & I $(42)$ & 2.89 & ps & 0.56 & 440 & 0.74 & $287+120$ & -6707.89 & Li I (1) & 0.00 & & 0.10: & 100: & - & - & $0.47 \mathrm{~b}$ \\
\hline
\end{tabular}


Table 12. Johnson $(U B V R)$ Photometric Data of BN Ori

\begin{tabular}{|c|c|c|c|c|c|}
\hline RJD \# & $V$ & $U-B$ & $B-V$ & $V-R$ & $\mathrm{~T}, \mathrm{O}$ \\
\hline 3135.3453 & 9.578 & 0.117 & 0.466 & 0.432 & $1, \mathrm{~S}$ \\
\hline 3154.4141 & 9.609 & 0.121 & 0.453 & & $1, \mathrm{~S}$ \\
\hline 3188.3667 & 9.666 & 0.132 & 0.465 & 0.399 & $1, \mathrm{~S}$ \\
\hline 3418.4326 & 9.601 & 0.133 & 0.456 & 0.413 & $1, \mathrm{~S}$ \\
\hline 3445.4423 & 9.612 & 0.142 & 0.503 & 0.455 & $1, \mathrm{~S}$ \\
\hline 3411.4312 & 9.598 & 0.137 & 0.500 & 0.432 & $1, \mathrm{~S}$ \\
\hline 3464.3878 & 9.623 & 0.140 & 0.521 & 0.453 & $1, \mathrm{~S}$ \\
\hline 3478.4121 & 9.611 & 0.154 & 0.503 & 0.435 & $1, \mathrm{~S}$ \\
\hline 3493.3998 & 9.606 & 0.154 & 0.489 & 0.498 & $1, \mathrm{~S}$ \\
\hline 3780.3889 & 9.612 & 0.163 & 0.448 & 0.456 & $1, \mathrm{~S}$ \\
\hline 3782.4112 & 9.609 & 0.143 & 0.501 & 0.456 & $1, \mathrm{~S}$ \\
\hline 3784.3987 & 9.599 & 0.138 & 0.511 & 0.458 & $1, \mathrm{~S}$ \\
\hline 3788.4356 & 9.623 & 0.144 & 0.546 & 0.453 & $1, \mathrm{~S}$ \\
\hline 3811.3765 & 9.632 & 0.148 & 0.564 & 0.533 & $1, \mathrm{~S}$ \\
\hline 3843.3876 & 9.607 & 0.139 & 0.554 & 0.467 & $1, \mathrm{~S}$ \\
\hline 3876.3978 & 9.600 & 0.127 & 0.476 & 0.476 & $1, \mathrm{~S}$ \\
\hline 5261.3245 & 9.598 & 0.143 & 0.465 & 0.408 & $1, \mathrm{~S}$ \\
\hline 5267.3987 & 9.646 & 0.182 & 0.455 & 0.465 & $1, \mathrm{~S}$ \\
\hline 5365.4432 & 9.656 & 0.167 & 0.456 & 0.521 & $1, \mathrm{~S}$ \\
\hline 5398.4312 & 9.654 & 0.156 & 0.465 & 0.432 & $1, \mathrm{~S}$ \\
\hline 5449.4532 & 9.674 & 0.197 & 0.476 & 0.460 & $1, \mathrm{~S}$ \\
\hline 5780.3246 & 9.666 & 0.155 & 0.456 & & $1, \mathrm{~S}$ \\
\hline 6047.2999 & 9.628 & 0.153 & 0.518 & 0.468 & $1, \mathrm{Y}$ \\
\hline 6052.3910 & 9.646 & 0.146 & 0.507 & 0.459 & $1, \mathrm{Y}$ \\
\hline 6055.2624 & 9.644 & 0.146 & 0.503 & 0.458 & $1, \mathrm{Y}$ \\
\hline 6060.2455 & 9.651 & 0.155 & 0.514 & 0.458 & $1, \mathrm{Y}$ \\
\hline 6061.2560 & 9.652 & 0.133 & 0.503 & 0.705 & $1, \mathrm{Y}$ \\
\hline 6293.4701 & 9.645 & 0.262 & 0.420 & 0.493 & $3, \mathrm{G}$ \\
\hline 6298.4769 & 9.640 & 0.115 & 0.468 & 0.430 & $3, \mathrm{G}$ \\
\hline 6300.4735 & 9.692 & 0.177 & 0.479 & 0.503 & $3, \mathrm{G}$ \\
\hline 6308.4909 & 9.601 & 0.148 & 0.472 & 0.472 & $1, \mathrm{C}$ \\
\hline 6310.4623 & 9.666 & 0.136 & 0.462 & 0.463 & $1, \mathrm{C}$ \\
\hline 6315.4504 & 9.640 & 0.193 & 0.466 & 0.468 & $1, \mathrm{C}$ \\
\hline 6317.4803 & 9.648 & 0.116 & 0.476 & 0.449 & $1, \mathrm{C}$ \\
\hline 6319.4789 & 9.650 & 0.139 & 0.482 & 0.461 & $1, \mathrm{C}$ \\
\hline 6321.4769 & 9.610 & 0.147 & 0.471 & 0.468 & $1, \mathrm{C}$ \\
\hline 6323.4713 & 9.652 & 0.154 & 0.474 & 0.462 & $1, \mathrm{C}$ \\
\hline 6324.4423 & 9.629 & 0.146 & 0.480 & 0.470 & $1, \mathrm{C}$ \\
\hline 6328.4949 & 9.652 & 0.147 & 0.440 & 0.484 & $3, \mathrm{M}$ \\
\hline 6331.4356 & 9.643 & 0.135 & 0.441 & 0.461 & $3, \mathrm{M}$ \\
\hline 6333.4264 & 9.648 & 0.133 & 0.426 & 0.456 & $3, \mathrm{M}$ \\
\hline 6335.4276 & 9.653 & 0.094 & 0.467 & 0.445 & $3, \mathrm{M}$ \\
\hline 6338.3967 & 9.654 & 0.142 & 0.398 & 0.448 & $3, \mathrm{M}$ \\
\hline 6339.4466 & 9.649 & 0.119 & 0.453 & 0.437 & $3, \mathrm{M}$ \\
\hline 6340.3722 & 9.604 & 0.154 & 0.464 & 0.451 & $3, \mathrm{M}$ \\
\hline 6345.4571 & 9.695 & 0.168 & 0.461 & 0.459 & $3, \mathrm{M}$ \\
\hline 6347.4125 & 9.658 & 0.135 & 0.458 & 0.448 & $3, \mathrm{M}$ \\
\hline 6355.3875 & 9.654 & 0.135 & 0.441 & 0.454 & $3, \mathrm{M}$ \\
\hline 6358.3858 & 9.628 & 0.134 & 0.480 & 0.459 & $3, \mathrm{M}$ \\
\hline 6370.3468 & 9.675 & 0.052 & 0.442 & 0.500 & $3, \mathrm{M}$ \\
\hline 6375.3989 & 9.652 & 0.149 & 0.470 & 0.463 & $1, \mathrm{I}$ \\
\hline 6376.4511 & 9.641 & 0.131 & 0.510 & 0.434 & $1, \mathrm{I}$ \\
\hline 6396.4591 & 9.603 & 0.059 & 0.455 & 0.424 & $1, \mathrm{I}$ \\
\hline 6397.4574 & 9.654 & 0.103 & 0.478 & 0.483 & $1, \mathrm{I}$ \\
\hline 6399.3980 & 9.615 & 0.131 & 0.467 & 0.445 & $1, \mathrm{I}$ \\
\hline
\end{tabular}

Table 12. continued

\begin{tabular}{|c|c|c|c|c|c|}
\hline RJD \# & $V$ & $U-B$ & $B-V$ & $V-R$ & $\mathrm{~T}, \mathrm{O}$ \\
\hline 6680.4633 & 9.696 & 0.145 & 0.421 & 0.416 & $3, \mathrm{Y}$ \\
\hline 6681.4910 & 9.631 & 0.112 & 0.474 & 0.459 & $3, \mathrm{Y}$ \\
\hline 6682.4780 & 9.653 & 0.161 & 0.460 & 0.462 & $3, \mathrm{Y}$ \\
\hline 6683.4625 & 9.672 & 0.143 & 0.458 & 0.459 & $3, Y$ \\
\hline 6691.4998 & 9.609 & 0.174 & 0.478 & 0.447 & $3, \mathrm{Y}$ \\
\hline 6702.4133 & 9.685 & 0.150 & 0.451 & 0.454 & $3, \mathrm{Y}$ \\
\hline 6708.4101 & 9.694 & 0.197 & 0.451 & 0.473 & $3, \mathrm{Y}$ \\
\hline 6714.4086 & 9.678 & 0.126 & 0.480 & 0.469 & $3, \mathrm{Y}$ \\
\hline 6722.4158 & 9.640 & 0.129 & 0.455 & 0.465 & $3, Y$ \\
\hline 6730.3997 & 9.685 & 0.118 & 0.491 & 0.448 & $3, \mathrm{P}$ \\
\hline 8490.4883 & 9.615 & & 0.497 & 0.421 & $3, \mathrm{M}$ \\
\hline 8491.4728 & 9.646 & & 0.500 & 0.437 & $3, \mathrm{M}$ \\
\hline 8492.4814 & 9.637 & & 0.499 & 0.448 & $3, \mathrm{M}$ \\
\hline 8494.4881 & 9.634 & & 0.496 & 0.437 & $3, \mathrm{M}$ \\
\hline 8495.4806 & 9.647 & - & 0.479 & 0.467 & $3, \mathrm{M}$ \\
\hline 8496.4796 & 9.666 & $\longrightarrow$ & 0.486 & 0.462 & $3, \mathrm{M}$ \\
\hline 8498.4674 & 9.640 & 0.163 & 0.494 & 0.472 & $3, \mathrm{M}$ \\
\hline 8499.4777 & 9.668 & & 0.481 & 0.468 & $3, \mathrm{M}$ \\
\hline 8508.4373 & 9.670 & 0.166 & 0.476 & 0.455 & $3, \mathrm{G}$ \\
\hline 8509.4540 & 9.646 & 0.147 & 0.497 & 0.437 & $3, \mathrm{G}$ \\
\hline 8510.4423 & 9.639 & 0.141 & 0.498 & 0.430 & $3, \mathrm{G}$ \\
\hline 8511.4572 & 9.662 & 0.170 & 0.480 & 0.467 & $3, \mathrm{G}$ \\
\hline 8512.4271 & 9.653 & $\longrightarrow$ & 0.485 & 0.452 & $3, \mathrm{G}$ \\
\hline 8513.4342 & 9.647 & 0.148 & 0.486 & 0.461 & $3, \mathrm{G}$ \\
\hline 8514.4398 & 9.647 & 0.152 & 0.501 & 0.460 & $3, \mathrm{G}$ \\
\hline 8515.4488 & 9.666 & 0.148 & 0.487 & 0.461 & $3, \mathrm{G}$ \\
\hline 8516.3688 & 9.650 & 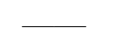 & 0.488 & 0.449 & $3, \mathrm{G}$ \\
\hline 8517.3853 & 9.644 & & 0.494 & 0.456 & $3, \mathrm{G}$ \\
\hline 8518.3947 & 9.657 & 0.148 & 0.493 & 0.459 & $3, \mathrm{G}$ \\
\hline 8519.4116 & 9.635 & 0.140 & 0.510 & 0.479 & $3, \mathrm{G}$ \\
\hline 8520.4186 & 9.614 & & 0.520 & 0.411 & $3, \mathrm{G}$ \\
\hline 8521.4207 & 9.665 & & 0.512 & 0.455 & $3, \mathrm{G}$ \\
\hline 8522.4230 & 9.617 & & 0.508 & 0.444 & $3, \mathrm{G}$ \\
\hline 8523.4076 & 9.640 & & 0.488 & 0.479 & $3, \mathrm{G}$ \\
\hline 8575.4053 & 9.642 & & 0.499 & 0.449 & $3, \mathrm{G}$ \\
\hline 8582.4644 & 9.627 & & 0.495 & 0.477 & $3, \mathrm{G}^{*}$ \\
\hline 8584.4290 & 9.648 & & 0.484 & 0.492 & $3, \mathrm{G}$ \\
\hline 8585.3857 & 9.635 & & 0.494 & 0.427 & $3, \mathrm{G}$ \\
\hline 8589.4612 & 9.644 & 0.090 & 0.477 & 0.438 & $3, \mathrm{G}$ \\
\hline 8871.4961 & 9.682 & 0.043 & 0.454 & 0.510 & $3, \mathrm{G}$ \\
\hline 8872.4633 & 9.700 & 0.052 & 0.475 & 0.505 & $3, \mathrm{G}$ \\
\hline 8873.4775 & 9.641 & 0.164 & 0.457 & 0.465 & $3, \mathrm{G}$ \\
\hline 8874.4310 & 9.674 & -.032 & 0.494 & 0.504 & $3, \mathrm{G}$ \\
\hline 8875.4425 & 9.669 & -.034 & 0.495 & 0.479 & $3, \mathrm{G}$ \\
\hline 8876.4102 & 9.692 & 0.041 & 0.479 & 0.508 & $3, \mathrm{G}$ \\
\hline 8877.4394 & 9.666 & -.042 & 0.486 & 0.487 & $3, \mathrm{G}$ \\
\hline 8878.4343 & 9.695 & -.072 & 0.463 & 0.508 & $3, \mathrm{G}$ \\
\hline 8879.4471 & 9.685 & 0.089 & 0.443 & 0.437 & $3, \mathrm{G}$ \\
\hline 8880.4245 & 9.679 & 0.031 & 0.487 & 0.493 & $3, \mathrm{G}$ \\
\hline 8881.4404 & 9.698 & 0.216 & 0.498 & 0.445 & $3, \mathrm{G}$ \\
\hline 8882.3933 & 9.660 & 0.010 & 0.488 & 0.478 & $3, \mathrm{G}$ \\
\hline 8883.3891 & 9.668 & 0.041 & 0.485 & 0.503 & $3, \mathrm{G}$ \\
\hline 8885.4966 & 9.680 & 0.019 & 0.463 & 0.498 & $3, \mathrm{G}$ \\
\hline 8886.4654 & 9.701 & 0.047 & 0.472 & 0.471 & $3, \mathrm{G}$ \\
\hline 8888.4503 & 9.686 & 0.027 & 0.470 & 0.474 & $3, \mathrm{G}$ \\
\hline
\end{tabular}


Table 12. continued

\begin{tabular}{|c|c|c|c|c|c|}
\hline RJD \# & $V$ & $U-B$ & $B-V$ & $V-R$ & $\mathrm{~T}, \mathrm{O}$ \\
\hline 8890.4338 & 9.690 & 0.037 & 0.468 & 0.505 & $3, \mathrm{G}$ \\
\hline 8901.4808 & 9.651 & & 0.486 & 0.474 & $3, \mathrm{M}$ \\
\hline 8905.4986 & 9.644 & & 0.487 & 0.462 & $3, \mathrm{M}$ \\
\hline 8906.4914 & 9.625 & & 0.478 & 0.449 & $3, \mathrm{M}$ \\
\hline 8907.4664 & 9.677 & & 0.467 & 0.472 & $3, \mathrm{M}$ \\
\hline 8914.4577 & 9.641 & & 0.483 & 0.453 & $3, \mathrm{M}$ \\
\hline 8915.4545 & 9.625 & $\longrightarrow$ & 0.494 & 0.441 & $3, \mathrm{M}$ \\
\hline 8920.4546 & 9.639 & 0.156 & 0.497 & 0.446 & $3, \mathrm{M}$ \\
\hline 8922.4626 & 9.655 & 0.146 & 0.480 & 0.440 & $3, \mathrm{M}$ \\
\hline 8923.4692 & 9.647 & & 0.499 & 0.454 & $3, \mathrm{M}$ \\
\hline 8925.4663 & 9.634 & & 0.505 & 0.450 & $3, \mathrm{M}$ \\
\hline 8927.4360 & 9.632 & & 0.491 & 0.469 & $3, \mathrm{M}$ \\
\hline 8954.4840 & 9.718 & & 0.476 & 0.481 & $3, \mathrm{G}$ \\
\hline 9234.4364 & 9.685 & & 0.476 & 0.480 & $3, \mathrm{G}$ \\
\hline 9235.4589 & 9.690 & & 0.471 & 0.469 & $3, \mathrm{G}$ \\
\hline 9238.4258 & 9.692 & - & 0.458 & 0.481 & $3, \mathrm{G}$ \\
\hline 9239.4933 & 9.697 & 0.049 & 0.472 & 0.481 & $3, \mathrm{G}$ \\
\hline 9259.5155 & 9.650 & & 0.487 & 0.457 & $3, \mathrm{M}$ \\
\hline 9260.4928 & 9.631 & & 0.485 & 0.455 & $3, \mathrm{M}$ \\
\hline 9262.5115 & 9.654 & & 0.483 & 0.452 & $3, \mathrm{M}$ \\
\hline 9264.5126 & 9.655 & & 0.484 & 0.450 & $3, \mathrm{M}$ \\
\hline 9271.4970 & 9.654 & & 0.495 & 0.447 & $3, \mathrm{M}$ \\
\hline 9274.4727 & 9.661 & & 0.478 & 0.455 & $3, \mathrm{M}$ \\
\hline 9286.4853 & 9.640 & & 0.499 & 0.446 & $3, \mathrm{M}$ \\
\hline 9615.4277 & 9.649 & 0.167 & 0.470 & 0.468 & $3, \mathrm{G}$ \\
\hline 9619.4415 & 9.638 & & 0.496 & 0.468 & $3, \mathrm{G}$ \\
\hline 9620.4566 & 9.644 & 0.155 & 0.503 & 0.466 & $3, \mathrm{G}$ \\
\hline 9621.4657 & 9.646 & 0.134 & 0.485 & 0.476 & $3, \mathrm{G}$ \\
\hline 9622.4624 & 9.626 & 0.158 & 0.503 & 0.450 & $3, \mathrm{G}$ \\
\hline 9948.4587 & 9.676 & & 0.468 & 0.502 & $3, \mathrm{G}$ \\
\hline 9950.4549 & 9.724 & & 0.480 & 0.514 & $3, \mathrm{G}$ \\
\hline 9952.4697 & 9.700 & & 0.476 & 0.488 & $3, \mathrm{G}$ \\
\hline 9954.4556 & 9.623 & 0.157 & 0.501 & 0.443 & $3, \mathrm{G}$ \\
\hline 9956.4432 & 9.661 & 0.155 & 0.503 & 0.480 & $3, \mathrm{G}$ \\
\hline 9958.4530 & 9.664 & 0.143 & 0.466 & 0.459 & $3, \mathrm{G}$ \\
\hline 9960.4466 & 9.648 & 0.146 & 0.490 & 0.468 & $3, \mathrm{G}$ \\
\hline 9963.4463 & 9.623 & 0.150 & 0.497 & 0.444 & $3, \mathrm{G}$ \\
\hline 9965.4696 & 9.668 & 0.146 & 0.489 & 0.456 & $3, \mathrm{G}$ \\
\hline 9968.4461 & 9.656 & 0.147 & 0.472 & 0.459 & $3, \mathrm{G}$ \\
\hline 9970.4159 & 9.628 & 0.158 & 0.486 & 0.477 & $3, \mathrm{G}$ \\
\hline 9973.3948 & 9.642 & 0.202 & 0.467 & 0.477 & $3, \mathrm{G}$ \\
\hline 9975.4000 & 9.633 & 0.139 & 0.493 & 0.457 & $3, \mathrm{G}$ \\
\hline 9978.3992 & 9.655 & 0.161 & 0.481 & 0.438 & $3, \mathrm{G}$ \\
\hline 9984.4238 & 9.646 & 0.140 & 0.488 & 0.453 & $3, \mathrm{G}$ \\
\hline 9987.4242 & 9.670 & 0.117 & 0.487 & 0.473 & $3, \mathrm{G}$ \\
\hline \multicolumn{3}{|c|}{ \# JD-2440000 } & \multicolumn{3}{|c|}{$\underline{\text { ROTOR observers }}$} \\
\hline \multirow{4}{*}{\multicolumn{3}{|c|}{$\begin{array}{l}\text { * simultaneous with the } \\
\text { high-resolution spectrum } \\
\text { from SAO (Sect. } 4.3 \text { ) }\end{array}$}} & $\mathrm{C}:$ & \\
\hline & & & G: & \multicolumn{2}{|c|}{ K.N. Grankin } \\
\hline & & & $\mathrm{I}:$ & \multicolumn{2}{|c|}{ M.A. Ibragimov } \\
\hline & & & M: & \multicolumn{2}{|c|}{ S.Yu Melnikov } \\
\hline \multicolumn{3}{|c|}{$\underline{\text { Telescopes }}$} & $\mathrm{P}:$ & \multicolumn{2}{|c|}{ D.L. Portnov } \\
\hline \multicolumn{3}{|c|}{ 1: Zeiss 600 reflector $(60 \mathrm{~cm})$} & S: & \multicolumn{2}{|c|}{ V.S. Shevchenko } \\
\hline \multicolumn{3}{|c|}{ 3: AZT 14 reflector $(48 \mathrm{~cm})$} & $\mathrm{Y}:$ & \multicolumn{2}{|c|}{ S.D. Yakubov } \\
\hline
\end{tabular}

